\title{
Delayed brain sensitivity to the same face features in ageing during gender and emotion categorisation tasks
}

\author{
Rory Boyle
}

Student number: 2224840B

Supervisor: Dr. Guillaume Rousselet 


\section{Abstract}

An age-related slowing in face processing has been reported but it remains unclear whether this slowing affects the facial information that is processed in the brain, and relied upon for making socially relevant decisions.

Using 'Bubbles' and electroencephalography (EEG), the effect of ageing on the information content of brain activity during tasks requiring emotion and gender categorisation of realworld faces was investigated.

Both older and younger adults relied upon mouth visibility for accurate emotion recognition, and eye visibility for accurate gender recognition. However, the reliance upon eye visibility for gender recognition was stronger in the older adult. Both groups displayed greatest brain sensitivity to the same task-relevant information (eyes and mouth), but the processing of this information appeared to be delayed in the older adult.

These findings suggest that the age-related delay and decline in face processing is due to a slower processing of task-relevant information, instead of a change in the information that is processed. As such, halting the slowing of visual information processing may be a promising target for improving face processing performance in older adults. 


\section{Author Summary}

Ageing impairs and delays the processing of faces, causing problems in social interaction for the elderly. However, it is unclear whether the delay in processing a face impacts the information that is processed.

We recorded the brain activity of an old and young group while they viewed faces and decided whether they were male or female, and whether they were happy or neutral. We covered the faces in a mask, through which only some parts of the face were visible. This allowed us to identify what parts of the face both groups used to make decisions, and what parts of the face drove brain activity.

We found that the older participant was more reliant on using the eye to determine gender, but both groups used the mouth to determine emotion. We found that brain activity was mostly driven by the mouth and eye in both groups, but was delayed in response to these features in the older group. This suggests that the information that is processed in the brain by older adults is not different compared to younger adults, but instead takes longer to process. Future research might investigate whether the delayed processing in older adults can be prevented. 


\section{Introduction}

Ageing causes slower and less accurate face processing (Grady et al., 1994; Gunning-Dixon et al., 2003). This impairs judgements of emotional expression (Ruffman et al., 2008), an important component of social communication (Haxby et al., 2002). Consequently, older adults might have poorer social interactions, contributing to loneliness, with negative effects for mental health (Santini et al., 2016).

Cognitive ageing explanations of the face processing decline include a reduced specificity of the visual cortex for face processing (Voss et al., 2008), increased prefrontal recruitment to compensate for reduced visual cortex activity (Grady, 2008), and a reduced ability to ignore task-irrelevant information (Gazzaley et al., 2008). Another explanation is the age-related slowing of 1 millisecond per year in face processing (Rousselet et al., 2010; Bieniek et al., 2013). However, it is unclear whether this delay has functional consequences or represents superficial changes in the shape of brain activity.

'Bubbles' and EEG can be used to investigate whether this delay affects the information content of visual brain activity. 'Bubbles' covers face images in a mask containing randomly located Gaussian apertures which reveal some image information (Gosselin \& Schyns, 2001). This technique has shown a reliance on the mouth for judging whether a face is happy or neutral and on the eyes for judging gender (Gosselin \& Schyns, 2001; Schyns et al., 2002) and that an early event-related potential (ERP), the N170, likely reflects an automatic brain response to the contralateral eye, at least in emotion and gender categorisation tasks (Smith et al., 2004). It has also found that another ERP, the P300, is modulated by the same information relied upon for judgements, i.e. the mouth for emotional expression (Smith et al., 2004). 
Jaworska et al. (2016) reported that younger and older adults relied on eye visibility for fast and accurate face detection, although older adults had a stronger reliance. The brain response to faces was driven by the presence of the contralateral eye, which was processed 40 milliseconds later in older adults (Jaworska et al., 2016). The association between contralateral eye visibility and brain response was weaker in older adults, suggesting a weaker and delayed brain response to the eyes, despite a stronger reliance on eye visibility for accurate performance (Jaworska et al., 2016).

It remains unclear whether these findings can be extended to socially relevant tasks (i.e. gender and emotion categorisation), as age differences have only been studied using face detection tasks (Rousselet et al., 2010; Jaworska et al., 2016). Furthermore, Bubbles studies have used oval-shaped, greyscale face images with normalised hairstyles, which are not representative of real-world faces.

The present study aims to extend previous findings by comparing older and younger adults in gender and emotion categorisation tasks using naturalistic face images and to investigate whether the facial information processed by the brain changes with age. Ageing is predicted to cause a greater reliance on the same task-relevant information as younger adults, along with delayed processing of this information and increased sensitivity to task-irrelevant information.

The hypothesis was partly supported as the older adult displayed a stronger reliance on the same task-relevant information relied up on by younger adults in a gender recognition task but not in an emotion recognition task, and displayed increased sensitivity to task-irrelevant information in the left, but not the right, hemisphere for both tasks. Furthermore, a delay in face processing was evident in the older adult. 


\section{Method Section}

\section{Participants}

Thirteen young adults and one older adult participated in the study. Table 1 contains basic participant information. Participants were excluded if they reported any eye condition, history of mental illness, psychotropic medication usage, neurological condition, head injuries (including concussion), or had not had their vision tested in the last 2 years (for young adults) or in the last year (older adult). Additionally, older participants were excluded if they had mild cognitive impairment as assessed using the Montreal Cognitive Assessment (Nasreddine et al., 2005). Visual acuity and contrast sensitivity were tested prior to beginning the first testing session using the Colenbrander Mixed Contrast Card Set and the Mars Letter Contrast Sensitivity test, respectively (table 1). All participants had normal or corrected to normal visual acuity and contrast sensitivity scores were within the normal range of contrast sensitivity for both age groups (Dougherty et al., 2005). Prior to the experiment, all participants read a study information sheet and signed an informed consent form. The project title was 'Judging Face Expression and Sex' and the study was approved by the local ethics committee at the College of Science and Engineering, University of Glasgow (approval no. 300150158). Participants were compensated $£ 6 / \mathrm{hr}$ for their participation.

Table 1. Participant demographic information and median visual acuity and contrast sensitivity scores.

\begin{tabular}{llllll}
\hline $\begin{array}{l}\text { Median age } \\
\text { (age range) }\end{array}$ & $\begin{array}{l}\text { No. of participants } \\
\text { (females, males) }\end{array}$ & $\begin{array}{l}\text { Median years of } \\
\text { education (range) }\end{array}$ & HC63 & LC63 & $\begin{array}{l}\text { Contrast } \\
\text { Sensitivity }\end{array}$ \\
\hline $23(19-25)$ & $13(9,4)$ & $16.5(14-19)$ & 105.5 & 96 & 1.82 \\
67 & $1(0,1)$ & 18 & 104 & 98 & 1.8 \\
\hline
\end{tabular}




\section{Stimuli}

Stimuli were comprised of 40 images of 10 male and 10 female faces, obtained from the MaxPlanck FACES database (Ebner et al., 2010). Each face provided 2 images, one displaying a happy expression and another a neutral expression. Images were front view photographs, pasted on a uniform grey background. Face identities in the images were young adults. Unlike previous studies (Rousselet et al., 2014; Smith et al., 2004), the images were coloured rather than greyscale, hairstyles were not altered, and the inter-feature distances within each face varied. Faces were delineated and adjusted to an average pupil height, and then cropped and resized, but with original variations in head size, inter-feature distances and hairstyles maintained. Stimuli had an amplitude spectrum set to the mean amplitude of all faces and all had the same mean pixel intensity. Stimuli were 253 x 294 pixels with a visual angle of $3.71^{\circ} \times 13.58^{\circ}$

\section{Bubbles}

Face images were overlaid with Bubble masks, consisting of a grey mask covering the face image, with image information revealed through randomly located Gaussian aperture (Fig. 1). The location of the Gaussian apertures (hereafter referred to as Bubbles) varied with each trial. Each trial contained 20 Bubbles $(\mathrm{sd}=15)$, which allowed visibility of some image information but not so much information that the task was trivial. 


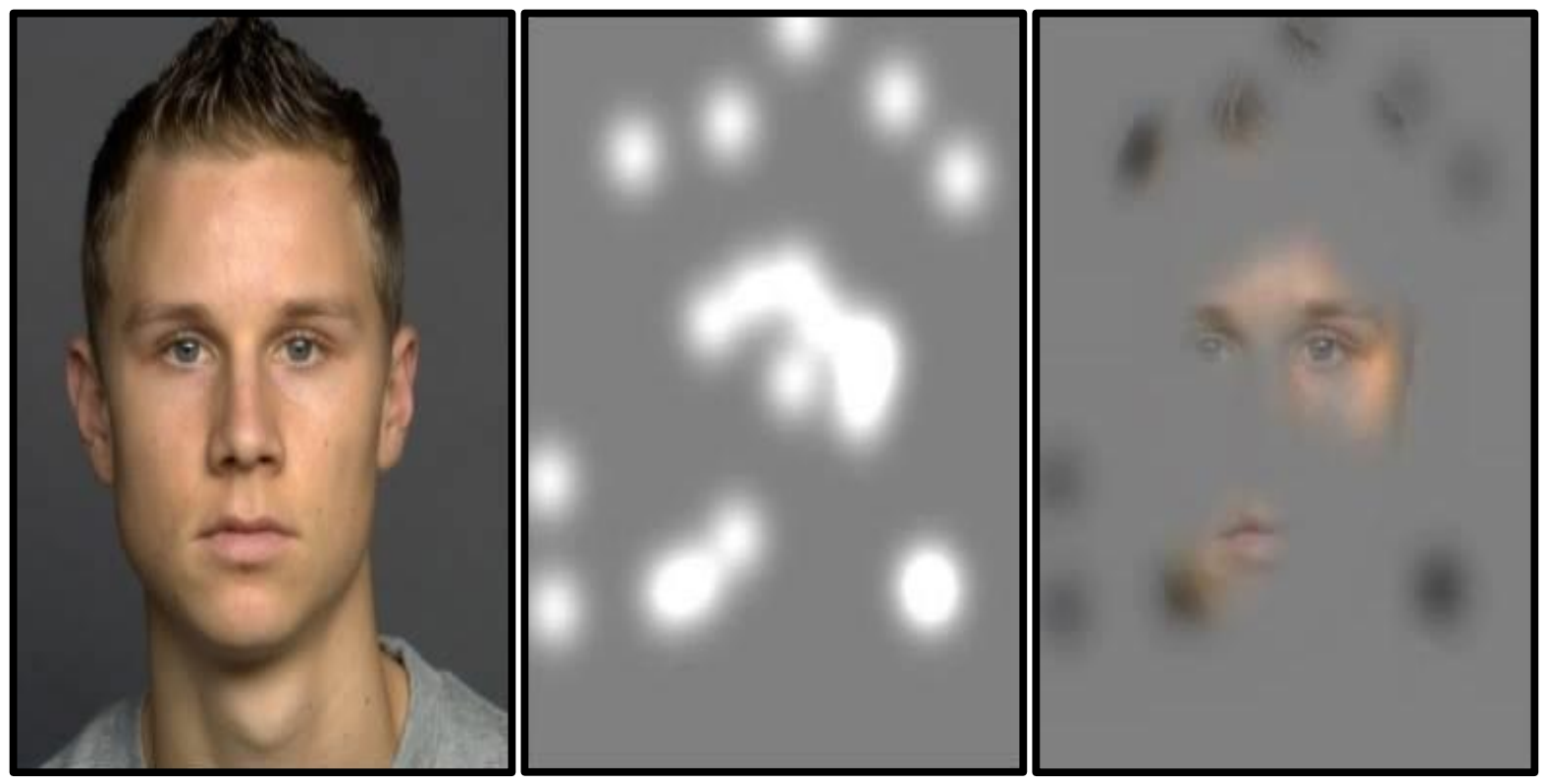

FIG. 1. Example of a bubble masked face stimulus used in the present study. In each trial, a face image (left) is overlaid with a bubble mask (centre), creating a stimulus (right) which is presented to the participant.

\section{Bubble-Warp}

In a typical Bubbles analysis, the same pixel location in different images reveals the same facial information. However, in the present study, because the natural variation in interfeature distances within each face was maintained, the same pixel location in different images could possibly have revealed slightly different facial information. For example, a Bubble on one image might have provided visibility of the eye, but the same Bubble (i.e. same location within the image) might have revealed visibility of the eyebrow in a different face. To control for the possible variance in the facial information revealed by the same Bubbles, the BubbleWarp technique (Gill et al., 2015) was used. This technique retrospectively warps the Bubble masks from each trial to an average face. The delineated co-ordinates of the face on each trial were then weighted by the degree of visibility afforded to the image by the Gaussian apertures in the Bubble mask. The weighting of these co-ordinates were then warped to an average face. 


\section{Experimental Procedure}

Before each experimental session, participants were fitted with an EEG cap and electrodes. Participants completed the experimental session while sitting in a dark, sound-attenuated booth, with their head resting on a chin rest, $50 \mathrm{~cm}$ from the monitor screen. Stimuli were displayed on a ViewPixx/EEG monitor (ViewPixx Technologies, Saint-Bruno, Canada).

Participants underwent two experimental sessions. In one session, participants performed a gender categorisation task, where they decided if each face image was male or female. Participants were instructed to press ' 1 ' or ' 2 ' on the numerical pad of the keyboard if they viewed a male or female face, respectively. In another session, participants performed an emotion categorisation task, where they decided if each image displayed a happy or a neutral expression. Participants were asked to press ' 1 ' if they viewed a happy face and ' 2 ' if they viewed a neutral face. The order of each session was counterbalanced across participants. Participants performed 15 blocks of 80 trials in each session. The first block of each session was a practice block where images were presented without bubble masks to familiarise participants with the stimuli and minimise spatial uncertainty. This was followed by 14 blocks of the images covered with Bubble masks. Participants were instructed to take a short break following the completion of each block, and to take a longer break if they felt necessary at the half-way point of the session. Each trial began with a small black fixation cross displayed in the centre of the monitor for a random duration of between $500-1000 \mathrm{~ms}$, followed by presentation of a face image covered with a bubble mask for $100 \mathrm{~ms}$. There was an interstimulus interval of $800 \mathrm{~ms}$. Each session lasted approximately 60 minutes, with an additional 60 minutes required to fit the EEG electrodes and ensure good signal quality. 


\section{EEG Recording}

EEG data were recorded at $512 \mathrm{~Hz}$ using a 128-channel BioSemi Active Two EEG system (BioSemi, Amsterdam, Netherlands). Four additional electrodes (UltraFlat Active BioSemi Electrodes) were placed at the outer canthi and below the eyes. Electrode offsets were kept between $\pm 20 \mu \mathrm{V}$.

\section{EEG Preprocessing}

EEG data were preprocessed using MATLAB 2013b and the open-source toolbox EEGLAB version 13_4_4b (Delorme \& Makeig, 2004). Data were first re-referenced off-line to an average reference and were then band-pass filtered between $1 \mathrm{~Hz}$ and $40 \mathrm{~Hz}$ using a noncausal fourth order Butterworth filter. This filtering technique can potentially distort onsets (Rousselet et al., 2014), but this was not a concern as onsets were not the focus of the present study. Data were epoched between -300 and 1000 ms around stimulus onset.

The dataset was then manually inspected on a participant-by-participant basis and noisy electrodes and noisy trials were removed. Independent component analysis (ICA) was then performed on the dataset in order to remove blink and eye-movement artefacts. Components representing blinks and eye movements were rejected (number of independent components removed: median $=7.5, \min =1, \max =16)$. After ICA, baseline correction was performed by removing the channel mean from each channel. Eye channels were then removed from the data. Following this, additional checks were performed to ensure removal of bad trials. First, data epochs with absolute threshold values larger than $100 \mu \mathrm{V}$ were removed. Secondly, data epochs containing linear trends with an absolute slope larger than $75 \mu \mathrm{V}$ per epoch and with an $\mathrm{R}^{2}$ value larger than 0.3 were removed. In all participants, the median number of trials available for analysis was, for gender task: $1069, \min =987, \max =1096$; for expression task: 1075.5, $\min =1043, \max =1094$. 
Finally, single-trial spherical spline current source density (CSD) waveforms were computed using the CSD toolbox (Kayser \& Tenke, 2006; Tenke \& Kayser, 2012) to sharpen ERP topographies and reduce the impact of volume-conducted activity. CSD waveforms were computed using 50 iterations and the parameters $\mathrm{m}=4$, lambda $=10^{-5}$.

\section{Electrode Selection}

The standard procedure in ERP and face processing studies is to analyse electrodes in the posterior lateral area of both hemispheres, which display the biggest N170 (i.e. amplitude difference compared to the reference electrode at around 150-220 ms) response to faces (Smith et al., 2007; Rousselet et al., 2014), and posterior midline electrodes, which are thought to be sensitive to perceptual decisions (Smith et al., 2007; Schyns et al., 2011). However, this procedure ignores early sensitivity to facial information in electrodes with a smaller amplitude difference than the electrode chosen for analysis.

To ensure the detection of early brain sensitivity to facial information, three electrode clusters were selected a priori to form three virtual electrodes (Fig. 2). Each virtual electrode contained the maximum mutual information, at each time point and each pixel, across the electrodes within that specific cluster. The three virtual electrodes were the left electrode (LE) which contained the left posterior lateral electrodes, the right electrode (RE) which contained the right posterior lateral electrodes and the central electrode (CE) which contained the posterior midline electrodes (Fig. 2). 


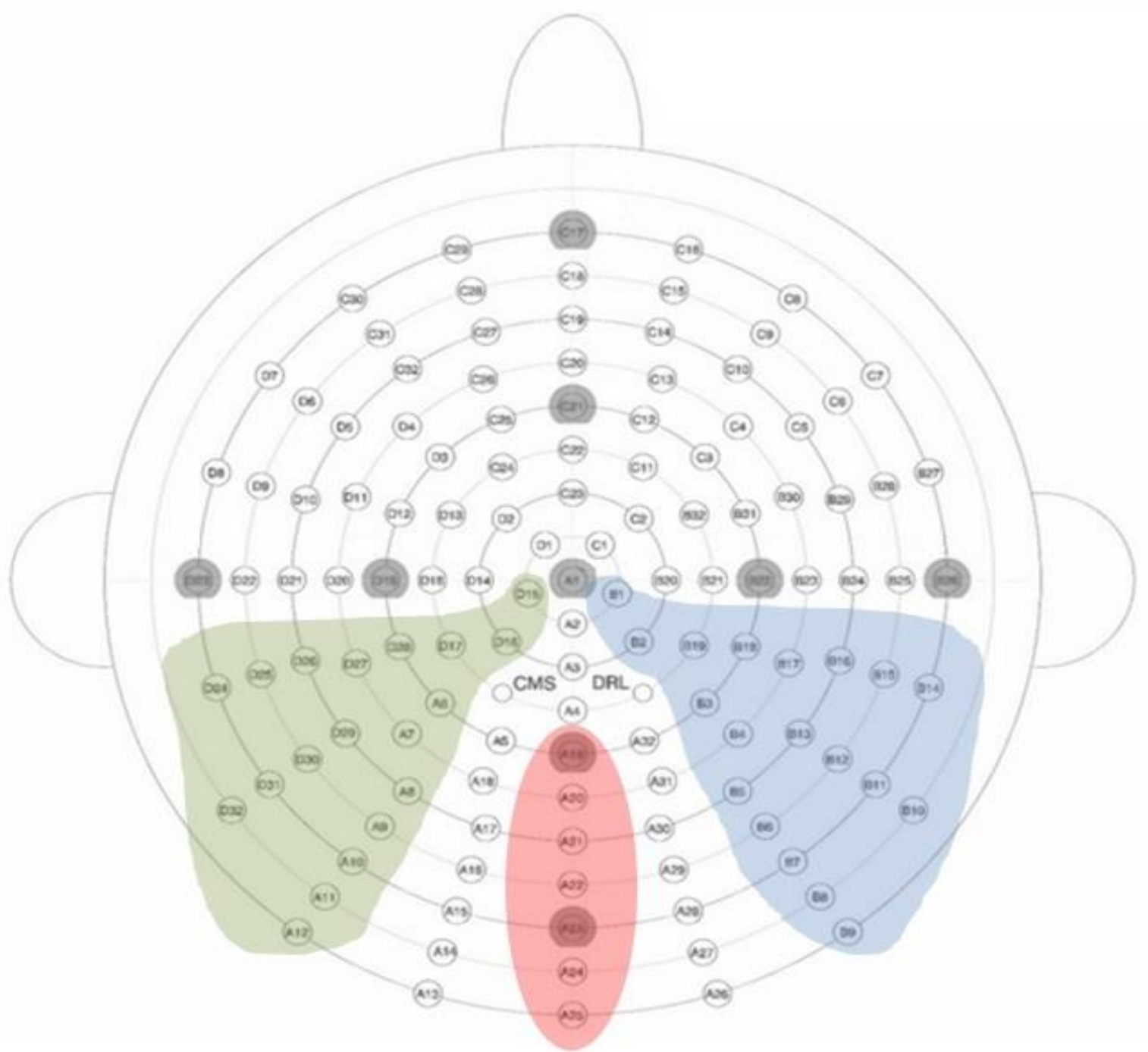

Fig. 2. Map of selected electrodes which formed the three virtual electrodes. LE is highlighted in green and contained electrodes D15-D32 and A6-A12. RE is highlighted in blue and contained electrodes B1-B19. CE is highlighted in red and contained electrodes A19-A25.

\section{Statistical Analysis}

\section{Analysis strategy}

Mutual information (MI) was used to analyse the data. MI was chosen because it is a nonparametric technique which quantifies the dependence between linear and/or non-linear variables on a common and meaningful scale (Ince et al., 2016). All median values reported are Harrell-Davis estimates of the median. 


\section{Single subject data analyses}

At the participant-level, MI quantified the dependence between pixel visibility and behavioural and EEG responses to the pixels. There were three variables of interest: MI(PIX, RT) measured, in bits, the strength of the association between pixel visibility and reaction times; MI(PIX, ACC) measured, in bits, the strength of the association between pixel visibility and response accuracy; MI(PIX, ERP) measured, in bits, the strength of the association between pixel visibility and single-trial ERPs.

\section{Group data analyses}

At the group-level, accuracy and median reaction time of the older participant was compared with the younger group by computing $95 \%$ confidence intervals of the median accuracy and reaction time of the younger group using the percentile bootstrap technique, with 1000 bootstrap samples.

Classification images of MI(PIX, RT) and MI(PIX, ACC) revealed the image pixels associated with modulations of behavioural response. Statistical significance of the MI(PIX, RT) and MI(PIX, ACC) values were established using a permutation max test, with 500 permutations and alpha level of 0.05, which corrected for multiple comparisons (Ince et al., 2016). Classification images of median MI(PIX, RT) and median MI(PIX, ACC) values for the young group were computed and compared with the classification images of the older participant.

MI(PIX, ERP) was calculated at each virtual electrode. For each electrode and task, the median of the maximum MI(PIX, ERP) in the young group was plotted across pixels and the timepoints 50 to $400 \mathrm{~ms}$. This was compared to time-courses of maximum MI(PIX, ERP) in the older adult. Classification images of the median of the maximum MI(PIX, ERP) across 
time and electrodes, for each electrode cluster and each task in the young group were compared to classification images of the maximum MI(PIX, ERP) for the older adult. 


\section{Results}

\section{Behavioural Performance}

For average behavioural performance, the older participant was less accurate in both tasks (gender $=70.9 \%$, expression $=75.6 \%)$ than the average performance of the young group $($ gender $=83.1 \%[79.1,86.4]$, expression $=89.7 \%[87.4,91])$. The older participant was slower in the gender task (660.6 ms) compared to the young group (536.6 ms [491.9, 615.9]), but was not significantly slower in the expression task $(585 \mathrm{~ms})$ compared to the young group (501.5 ms [471.2, 613.9]).

Task differences in both groups were similar, as both were less accurate in the gender task than in the expression task (old $=-4.7 \%$, young $=-4.9 \%[-9.5,-2.3]$ ). Both groups tended to respond faster in the gender task, an effect which was greater in the older participant, (old = $75.5 \mathrm{~ms}$ vs young $=13.2 \mathrm{~ms}[-47.3,71.2])$. 

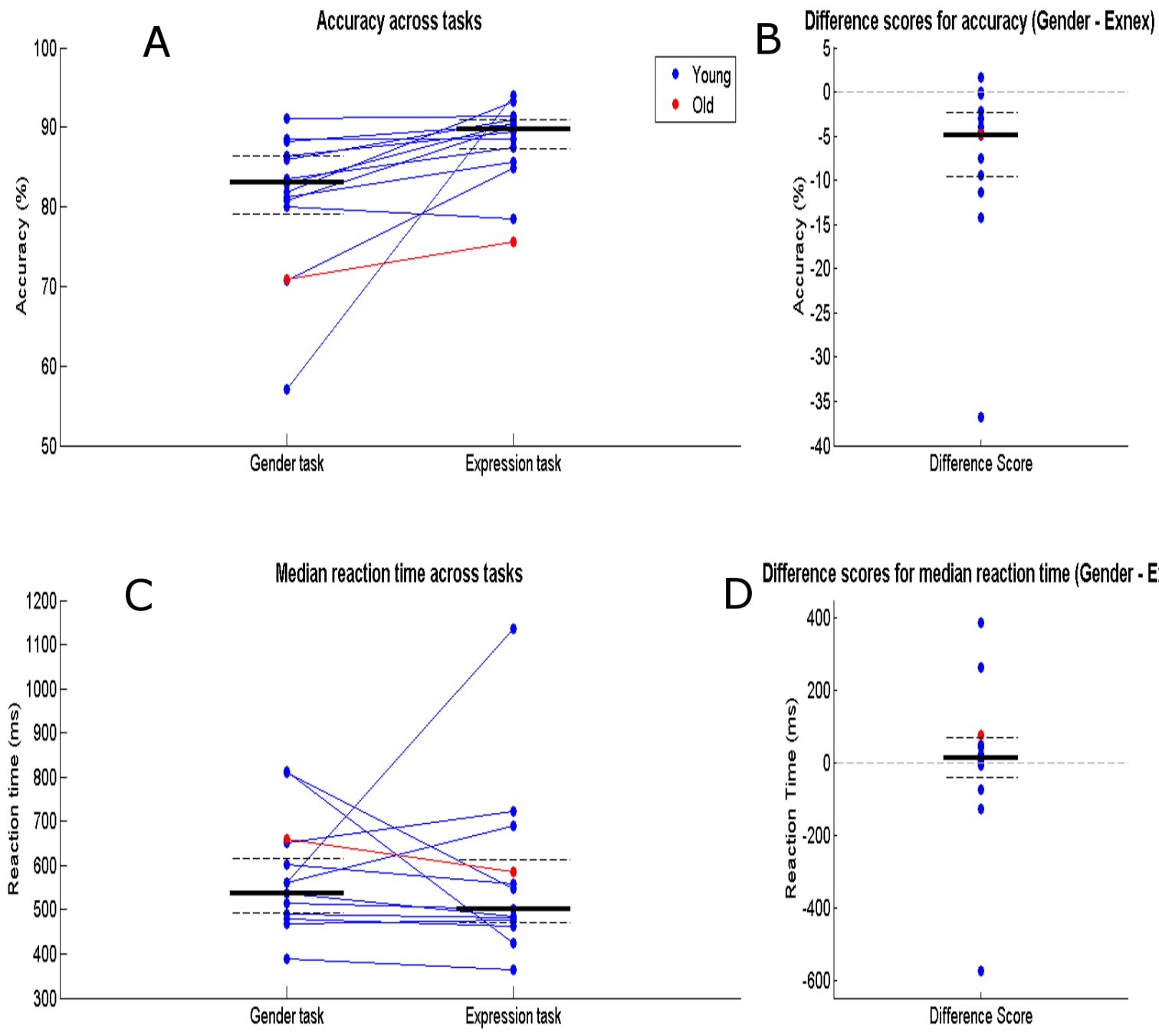

Difference scores for median reaction time (Gender - Exnex)

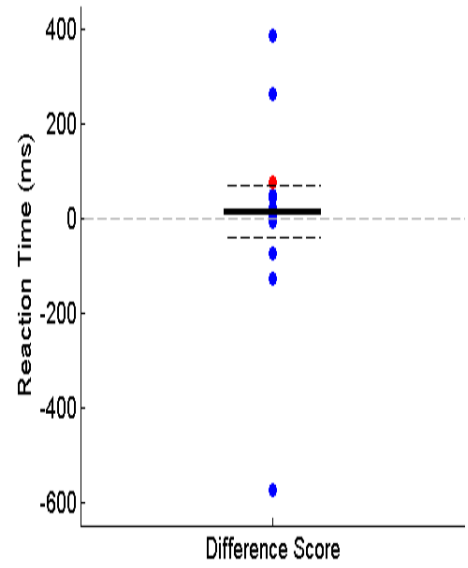

Fig. 3: Individual performance in accuracy and reaction across tasks and difference scores for all participants. 3A: Participant accuracy in each task is plotted with lines connecting a participant's accuracy in the gender task to their accuracy in the expression task. The median accuracy of young group plotted as a black line and 95\% confidence intervals plotted as two dashed black lines. 3B: Difference scores between tasks in accuracy for all participants with median and confidence intervals plotted as previously described. Difference scores were computed by subtracting each participant's accuracy in the expression task from their accuracy in the gender task. 3C: Median reaction time of individual participants in both tasks connected with lines. Median of median reaction time in the young group plotted as a black line and 95\% confidence intervals plotted as two dashed black lines. 3D: Difference scores between tasks in median reaction time. 
MI(PIX, ACC) and MI (PIX, RT)

Both groups displayed a reliance on eye visibility for accurate gender recognition, but not for fast responses, although this reliance was weaker and uncommon in the young group. Both groups displayed a strong reliance upon mouth visibility for fast and accurate emotion recognition.

Classification images of MI(PIX, ACC) for the gender task show that the older participant relied more heavily on eye visibility than the average participant in the young group (Fig. 4). MI(PIX, ACC) shows that associations between eye visibility and accuracy were weak and uncommon in the young group. A classification image showing this association in a younger participant is presented in figure 4 (all individual classification images are presented in supporting information, Fig. S1). MI(PIX, ACC) for the expression task reveal a strong reliance on mouth visibility for accurate responses across all participants (Fig. 4). MI(PIX, RT) for the gender task show that on average there was no strong reliance on eye visibility for fast responses in the young group (Fig. 4), although 3 participants did display a significant association between eye visibility and fast response. In the older participant, there was a weak but significant association between visibility of the centre of the hairline and fast responses. MI(PIX, RT) for the expression task show a strong and significant reliance on mouth visibility for fast responses in 12 participants (Fig. 4). In contrast, the older participant displayed a weak, and not significant, association between the visibility of the corner of the mouth and fast responses. 

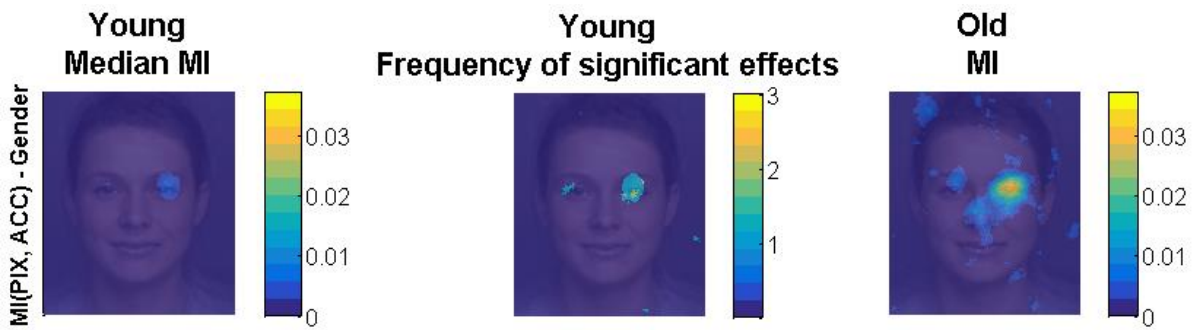

Old

Significant effects
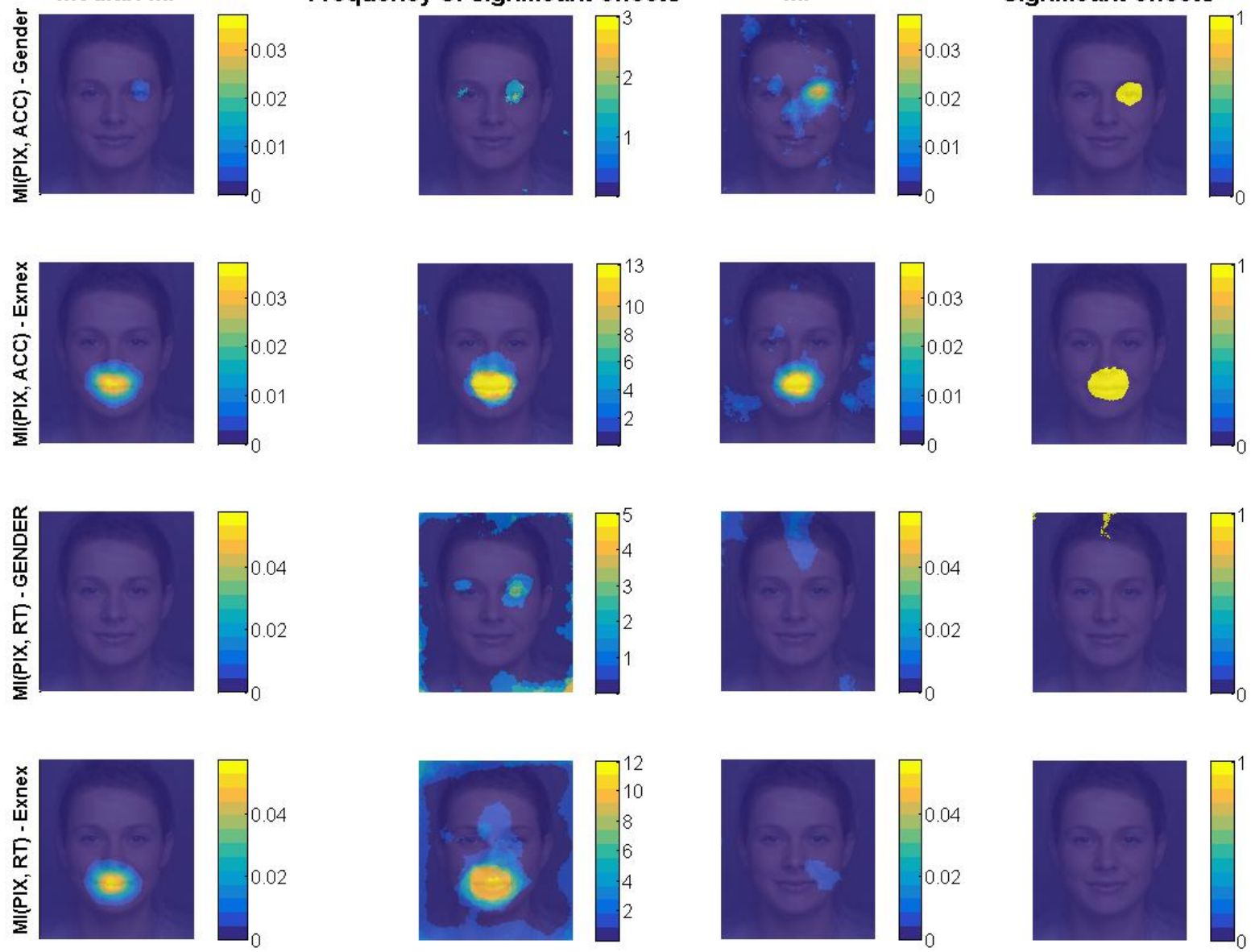

Fig. 4: Summary classification images of MI(PIX, ACC) and MI(PIX, RT) in younger adults compared to classification images of the older adult. The top two rows show classification images of MI(PIX, ACC) in the gender and expression task, respectively. The bottom two rows show MI(PIX, RT) in the gender and expression task, respectively. First column shows median MI of the young group for each measure. Second column shows the frequency of significant MI effects in the young group. Frequency of significant effects was established using a permutation max test with 500 permutations and alpha level of 0.05. Third column shows MI of the older participant for each measure, scaled to the maximum of the young group's median MI. Fourth column shows the presence of significant effects in the older participant. 

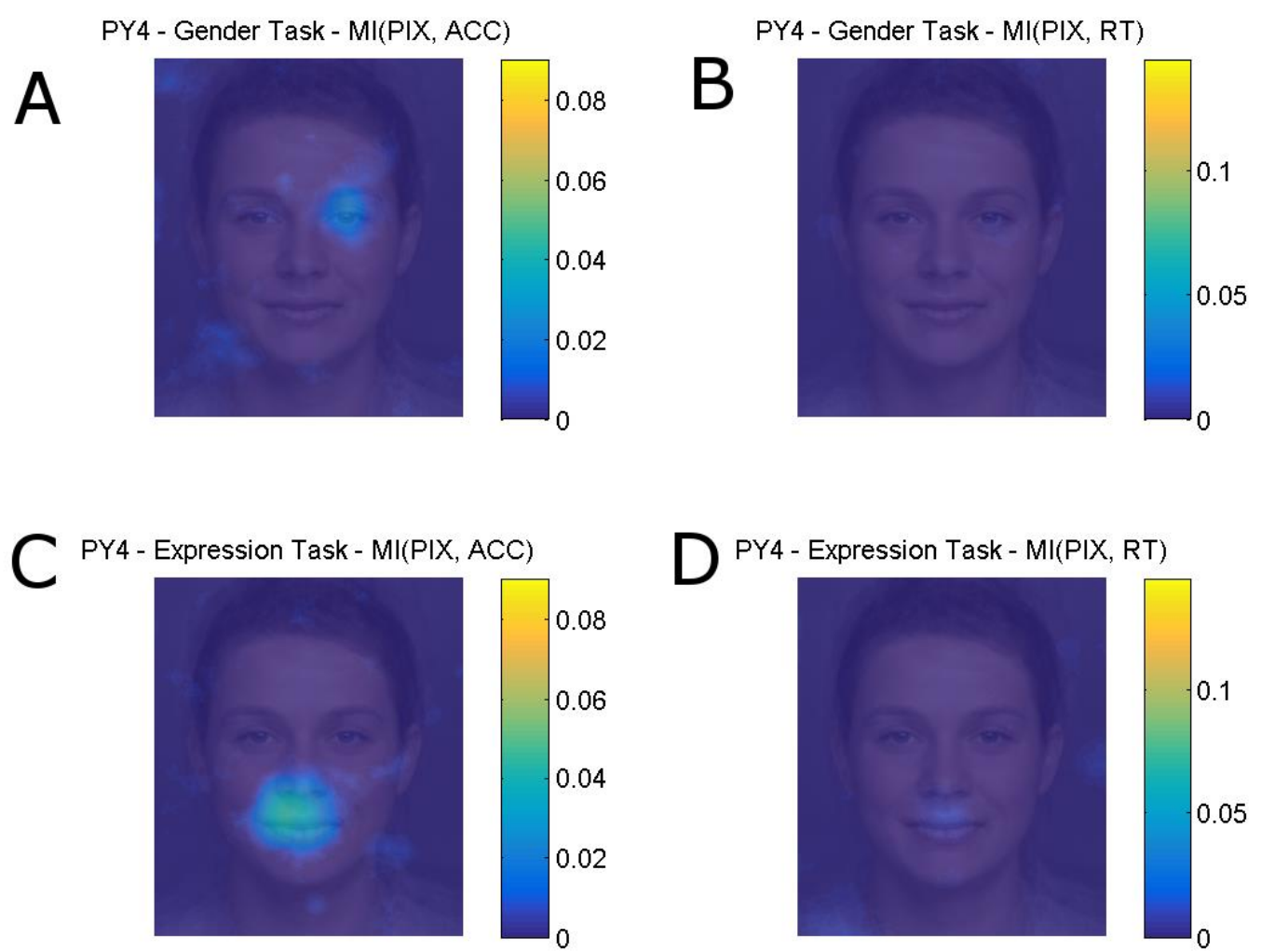

Fig. 5: Individual classification images of $M I(P I X, A C C)$ and $M I(P I X, R T)$ in a young adult (PY4). 5A: Association between eye visibility and accuracy in gender task. This association was uncommon across most young participants but was present in this participant. $5 B$ : Association between pixel visibility and fast responses in the gender task. No strong relationship between specific feature visibility and fast reaction time was observed. $5 C$ : Association between mouth visibility and accuracy in the expression task. 5D: Association between mouth visibility and fast responses in the expression task (MI is scaled down here but still indicates a strong association).

For MI(PIX, ACC) and MI(PIX, RT) in both tasks, the associations between pixel visibility and behaviour were weaker in the older participant. As such, classification images of these quantities, scaled to the maximum MI present in that quantity in the older participant were created (Fig. 6). This allows for closer detection of the features relied upon by the older participant for behaviour. MI(PIX, ACC) in the gender task at this scale shows the previously described reliance upon eye visibility but also reveals an association between nose and hairline visibility and accuracy. MI(PIX, ACC) in the expression task again reveals the reliance upon mouth visibility but also detects associations between visibility of pixels 
outside of the face and accuracy. MI(PIX, RT) in the gender task shows an association between hairline and also chin/jaw visibility and fast responses. MI(PIX, RT) in the expression task shows an association between cheek and mouth corner visibility and fast responses.
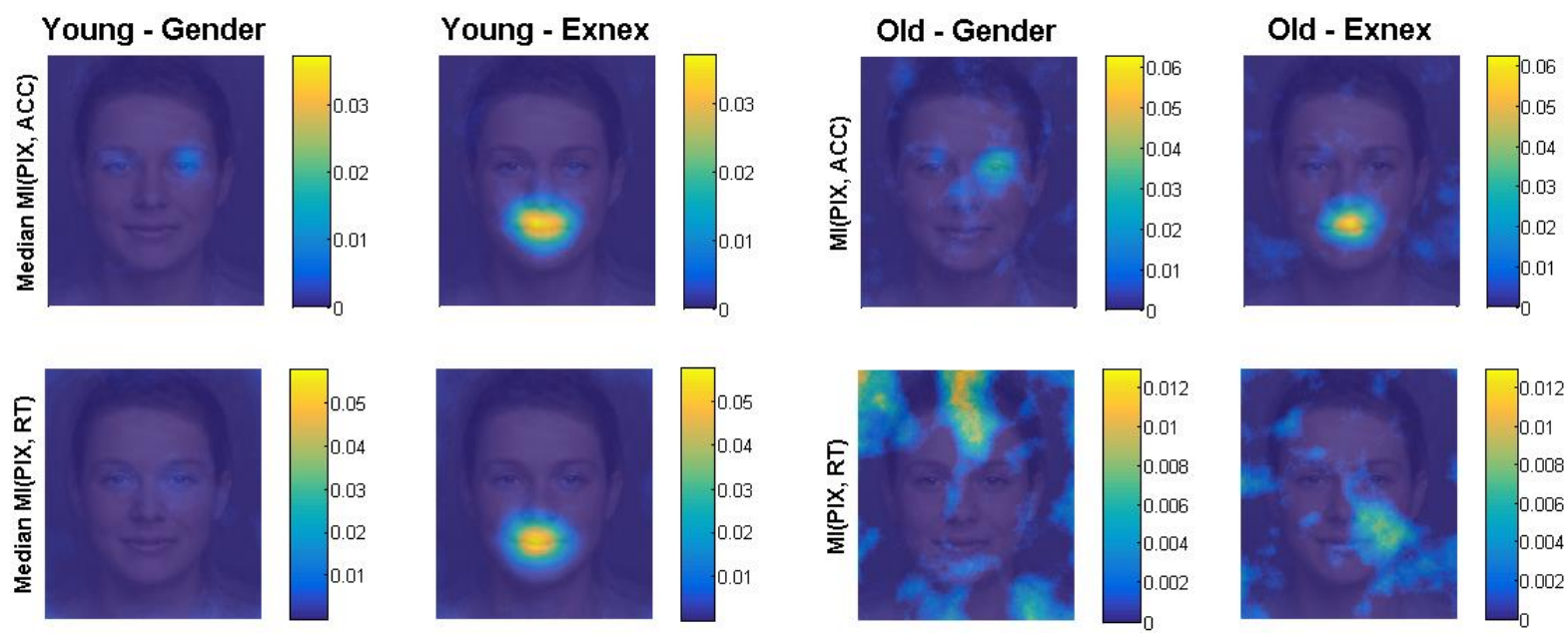

Fig. 6: Classification images of MI(PIX, ACC) and MI(PIX, RT) with scales adjusted for older adult. Top row shows MI(PIX, ACC) and bottom row shows MI(PIX, RT). First column shows median MI of young participants in the gender task. Second column shows median MI of young participants in the expression task. Third column shows MI of older participant in the gender task, with MI scaled according to the maximum MI of the older participant. Fourth column shows MI of older participant in the expression task with MI scaled according to the maximum MI of the older participant.

\section{MI(PIX, ERP)}

Across electrodes and tasks, both groups displayed greatest brain sensitivity to mouth visibility, with sensitivity to the eyes observed also. However, brain sensitivity was more varied in the older participant at the left electrode. MI peaks were delayed, but larger, in the older participant in comparison to the young group but no MI peak was observed in the older participant at the left electrode. MI(PIX, ERP) results for the posterior lateral electrodes are first presented, as they typically show greatest sensitivity to facial features such as contralateral eye (Rousselet et al., 2014). MI(PIX, ERP) results for the central electrode are 
then presented, which typically displays greatest sensitivity to perceptual decisions (Smith et al., 2007; Schyns et al., 2011).

\section{Left Electrode}

The time-course of MI(PIX, ERP) at the left electrode revealed that the older participant had weaker brain sensitivity to pixel visibility in both tasks, with a very early peak at around 120 ms in both tasks (Fig. 7). In contrast, the median time-course of MI(PIX, ERP) in the young group had a stronger peak at approximately $170 \mathrm{~ms}$. MI in the older participant was driven by a variety of features across timepoints, with greatest brain sensitivity to visibility of the ipsilateral eye, nose, contralateral jawline, and mouth in the gender task, and to visibility of the contralateral jawline, hairline and mouth in the expression task. MI in the young group was mostly modulated by mouth and contralateral eye visibility in both tasks, but with greatest sensitivity to mouth visibility.
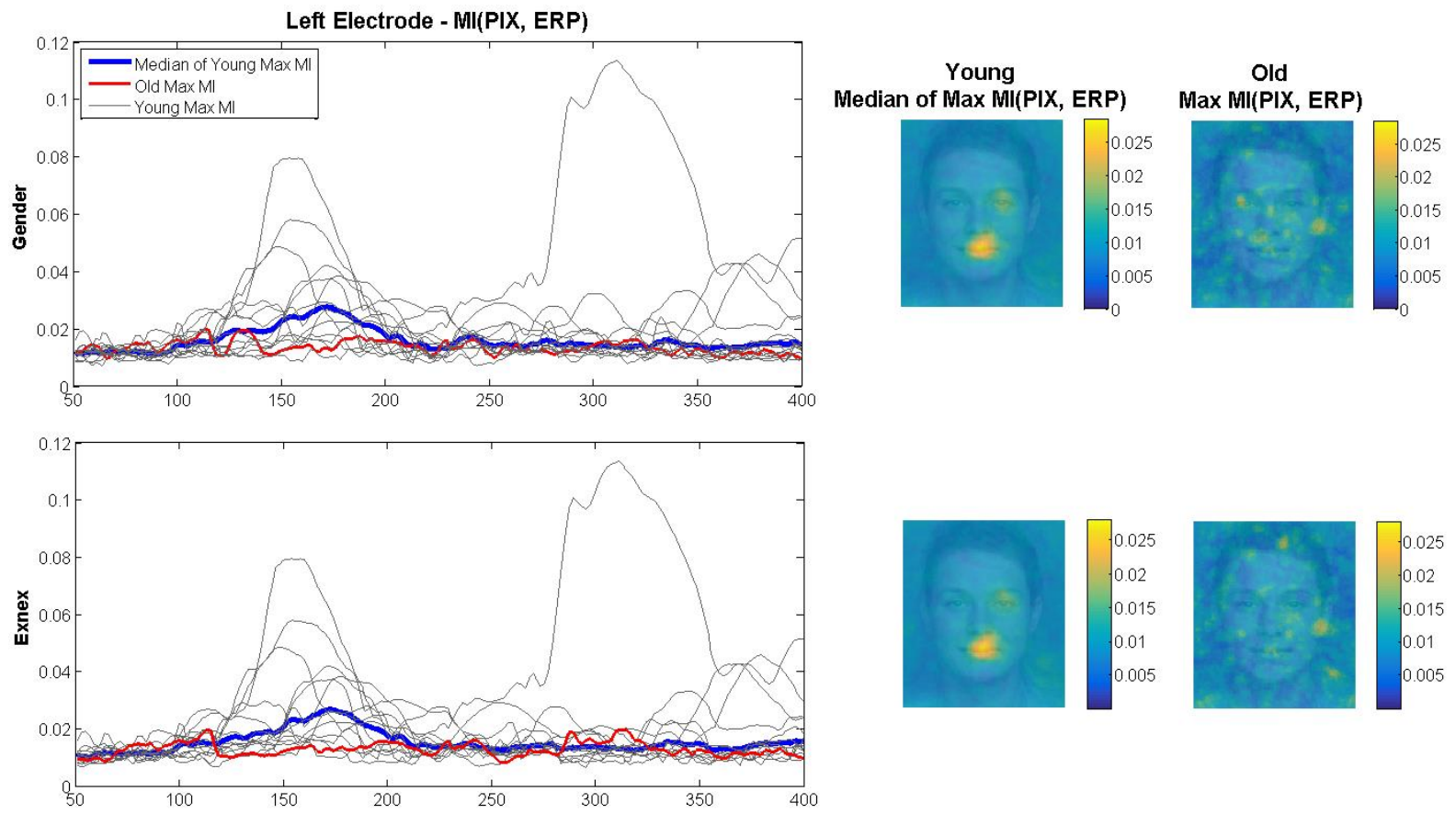

Fig. 7: Time-course plots and classification images of MI(PIX, ERP) at the left electrode. Top row shows MI(PIX, ERP) for gender task and bottom row shows MI(PIX, ERP) for expression task. Time-course plots show MI(PIX, ERP) across all timepoints and pixels. Young participant's individual maximum MI time-courses plotted in grey with the median of 
all young participant's maximum MI plotted in blue. The older participant's maximum MI time-course is plotted in red. Classification images show MI(PIX, ERP) in both tasks, per group, across all timepoints at the left electrode.

\section{Right Electrode}

The time-course of MI(PIX, ERP) at the right electrode revealed that the older participant had slightly stronger but delayed brain sensitivity to general pixel visibility in both tasks, with an early initial peak at around $110 \mathrm{~ms}$, followed by a later, larger, peak at around 205-210 ms, in both tasks (Fig. 8). In contrast, the median time-course of MI(PIX, ERP) in the young group had a peak at approximately $150 \mathrm{~ms}$. MI in both groups was modulated by mouth and contralateral eye visibility, with a greater sensitivity to mouth visibility relative to contralateral eye visibility in the young group.
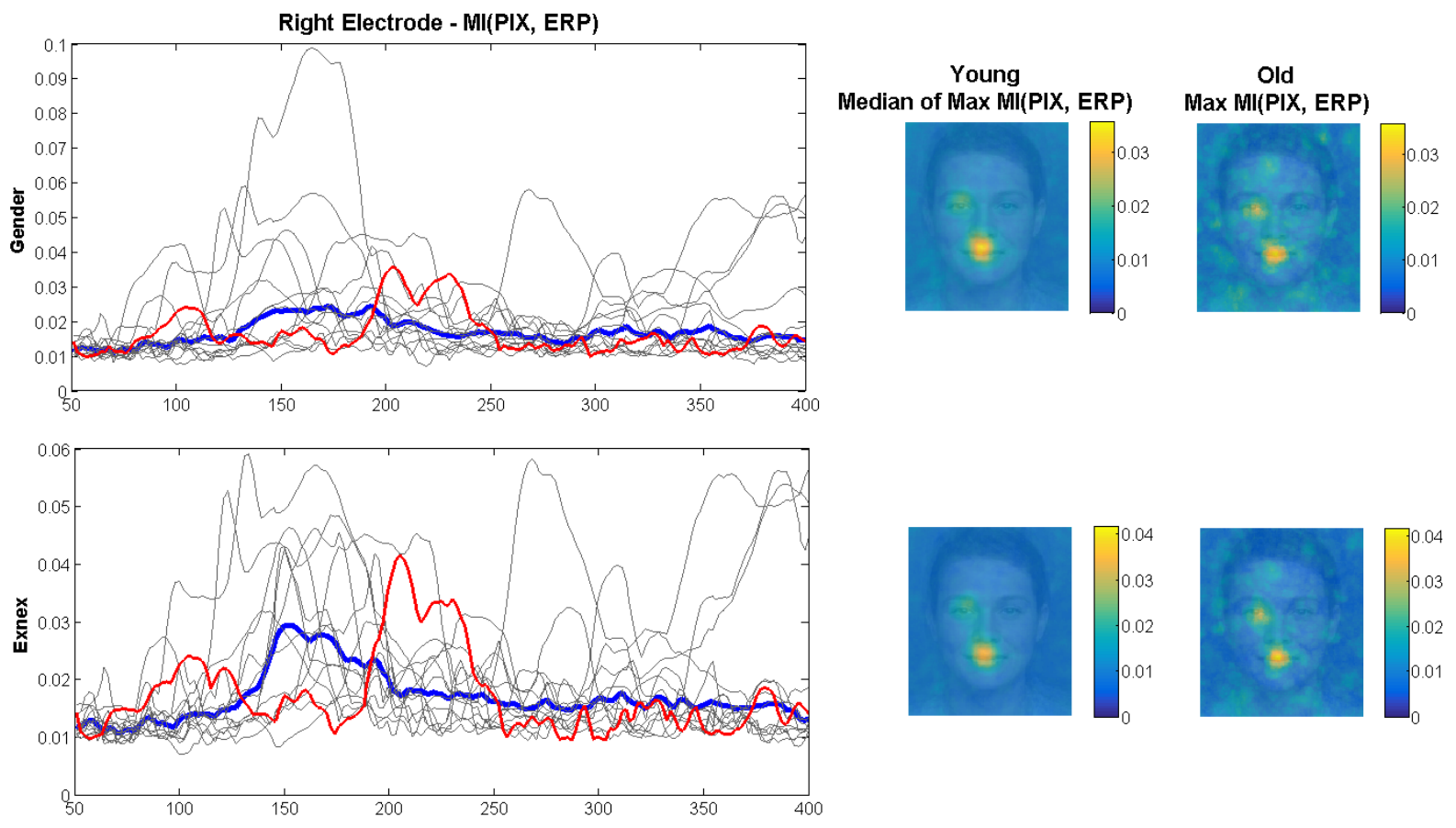

Fig. 8: Time-course plots and classification images of MI(PIX, ERP) at the right electrode.

Top row shows MI(PIX, ERP) for gender task and bottom row shows MI(PIX, ERP) for expression task. Time-course plots are scaled separately for each task due to the large maximum MI of a younger participant at around $175 \mathrm{~ms}$ in the gender task. However, median MI and old MI time-course peaks are located roughly around the same MI values. 


\section{Centre Electrode}

The time-course of MI(PIX, ERP) at the centre electrode revealed that the older participant had slightly stronger but delayed brain sensitivity to general pixel visibility in both tasks, with a very early peak at around $75 \mathrm{~ms}$, followed by a later peak at around $180 \mathrm{~ms}$, in both tasks (Fig. 8). In contrast, the median time-course of MI(PIX, ERP) in the young group had a peak at approximately $150 \mathrm{~ms}$. MI in the older participant was driven by a variety of features across timepoints, with greatest brain sensitivity to visibility of the mouth, nose and right eye in the gender task, and to visibility of the mouth and nose in the expression task. MI in the young group was modulated mainly by mouth visibility, with additional sensitivity to the right eye, in both tasks.
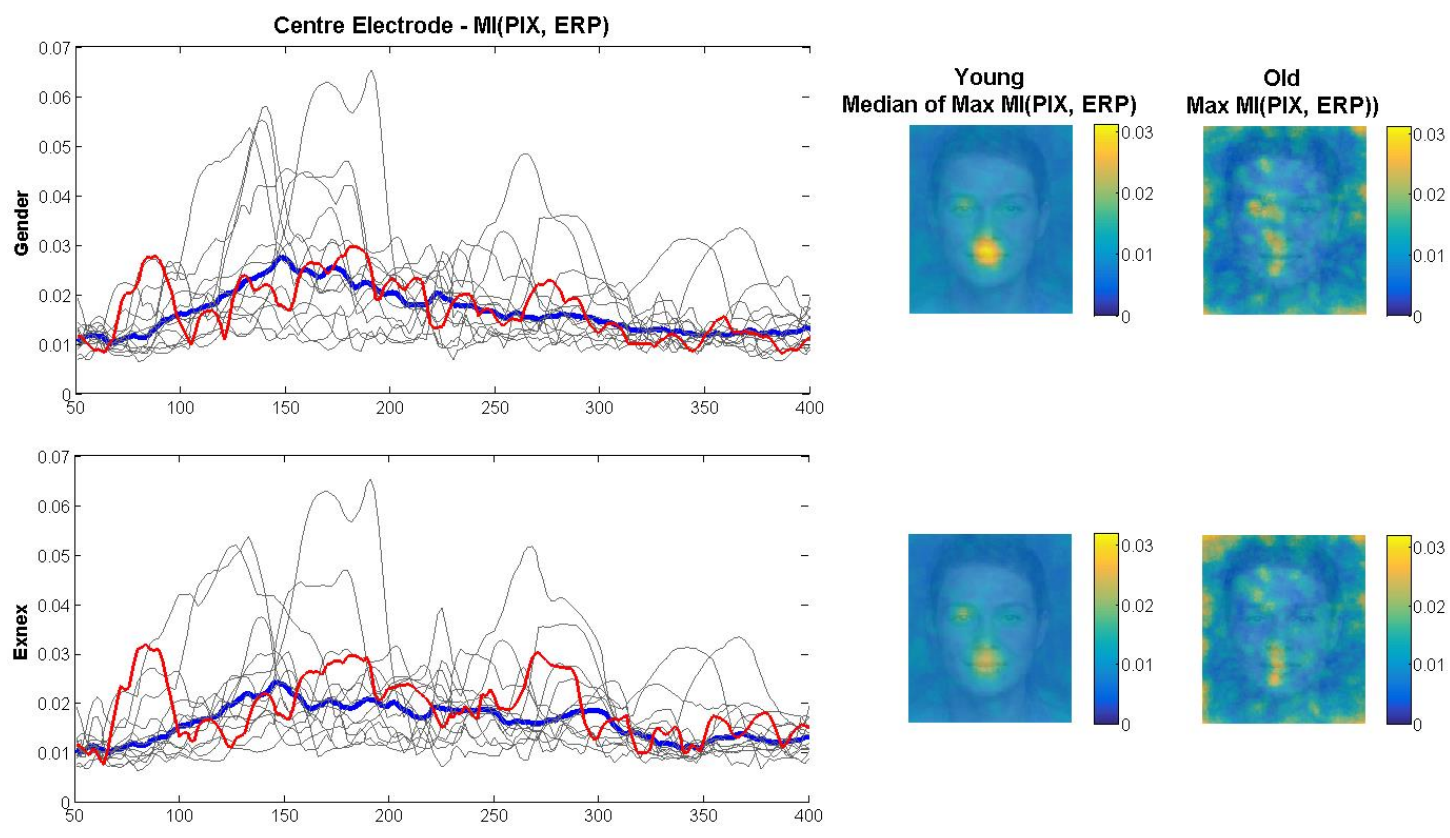

Fig. 9: Time-course plots and classification images of MI(PIX, ERP) at the centre electrode. Top row shows MI(PIX, ERP) for gender task and bottom row shows MI(PIX, ERP) for expression task. 


\section{Discussion}

The older participant was less accurate than the average performance of the young group when judging both gender and expression, which is supported by the evidence of negative ageing effects on different aspects of face processing performance, from emotion recognition (Sullivan et al., 2007) to face detection (Rousselet et al., 2010). Few studies have investigated age-related differences in face gender recognition, although Iidaka et al. (2002) reported no ageing effect when face stimuli were presented in full. As such, the present finding suggests a certain level of task difficulty is necessary to detect an ageing effect in gender recognition.

The present study supports the hypothesis of a stronger reliance in older adults on taskrelevant information, as reported by Jaworska et al. (2016) in a face detection task, but only in gender recognition and not emotion recognition. Furthermore, the association between eye visibility and accurate gender recognition, and mouth visibility and accurate emotion recognition (Gosselin \& Schyns, 2001; Schyns et al., 2002; Smith et al., 2004) was observed with naturalistic face images. However, no association between eye visibility and fast gender recognition was observed, in contrast to the findings reported by Smith et al. (2004). An explanation for this finding could be that gender recognition might be processed implicitly, as accurate gender recognition has been reported in conditions of near-absent attention (Reddy et al., 2004). As such, the greater range of information provided in the naturalistic face images (i.e. hairstyle, skin tone) may allow for more efficient implicit gender processing. In the greyscale, cropped images used by Smith et al. (2004), the sparser facial information might have resulted in implicit gender processing only when the eye was visible, thereby restricting fast responses to trials with eye visibility. In contrast, enough information may have been present in the naturalistic faces to allow fast implicit processing, regardless of eye visibility. 
Whereas previous studies have reported weaker MI in response to facial features in older adults (Jaworska et al., 2016), this effect was only evident in the left hemisphere, as the older adult displayed greater MI in response to facial features in the right hemisphere and posterior midline. However, it should be noted that MI from one older adult was compared to the median MI of the 13 young adults. As such, the possibility that the brain sensitivity of the older adult may not have been representative of brain sensitivity in the wider population of older adults must be considered. Moreover, by comparing MI in the older adult to the median MI of the younger group, the maximum MI of the younger group would be naturally reduced. Individual time-course plots of maximum MI in younger participants in right and central electrodes show a number of young adults with greater MI peaks compared to the older adult. (Fig. 8 and 9).

Brain sensitivity in both groups was greatest to task-relevant information. Again, this was not the case in the left hemisphere where brain sensitivity in the older participant was modulated by a wider range of facial information, such as contralateral jawline, ipsilateral eye, and nose as well as the mouth in the gender task, and by the contralateral jawline, hairline, and mouth in the expression task. This finding might suggest greater brain sensitivity in older adults to task-irrelevant information in the left hemisphere, which could lend support to the theory that the reduced ability of older adults to ignore task-irrelevant information underlies the age-related visual processing decline (Gazzaley et al., 2008). However, the present study did not establish the significance of MI values for the MI between ERP and pixel visibility. As a result, the statistical significance of the dependence between these two variables cannot be inferred, so it remains unclear whether brain sensitivity is in fact driven by these facial features in older adults.

Apart from the left hemisphere of the older adult, there were no differences observed in the brain sensitivity of either group to facial features between gender and emotion recognition 
tasks observed in the present study. This contrasts with previous evidence that brain sensitivity is driven mainly by the eyes in gender categorisation and the mouth in emotion categorisation (Smith et al., 2004). While early brain sensitivity to the contralateral eye is expected regardless of task (Smith et al., 2004), sensitivity to the mouth in the gender task is not typically reported. However, gender processing has been reported to have no effect on the N170 which suggests it is implicitly processed (Mouchetant-Rostaing et al., 2000;

Mouchetant-Rostaing \& Giard, 2003). As such, brain sensitivity to the same features could be expected, regardless of task.

The present study provides evidence that the reported age-related delay in face processing (Rousselet et al., 2010; Bieniek et al., 2013; Jaworska et al., 2016) exists for naturalistic faces. These findings suggest that the age-related delay in face processing is likely due to a slower processing of task-relevant information, instead of a change in the information content of visual brain activity as the older adult displayed sensitivity to mostly the same features as the younger adults. This finding is significant because it confirms a well-established phenomenon in a more socially relevant paradigm (i.e. with naturalistic stimuli and socially relevant tasks). As such, it could be worthwhile to investigate methods of attenuating this delay in order to counteract the age-related decline in face processing and by extension social communication in older adults.

However, in contrast to previous studies, this delay was observed in right posterior lateral and posterior midline regions of the brain, but not the left posterior lateral area. The lack of an observed delay in the left hemisphere could be explained by the observed lack of significant brain sensitivity to facial features in this region, as without a significant peak in MI, a delay in face processing would not have been visible. 
The findings of the present study, while valuable, are limited when linking brain sensitivity to behaviour as the mutual information between ERPs and behaviour was not investigated. Consequently, it is not possible to infer whether brain sensitivity to specific features in either task was also associated with the brain activity underlying fast and accurate gender and emotion recognition.

Further research is needed to establish whether the findings of the present study can be generalised to a larger sample of older adults, or whether they might instead reflect an individual difference of the older adult studied here. Moreover, in such a study, the statistical significance of the MI values between ERPs and pixel visibility should be calculated using permutation tests, which will allow the frequency of significant effects between these two variables to be established. Furthermore, the MI between ERPs and behavioural performance should be analysed in order to examine whether the brain sensitivity to specific facial features is also associated with fast and accurate behavioural performance. 


\section{Acknowledgements}

I would like to thank Dr. Guillaume Rousselet for his valuable advice and feedback during this project, Hannah Gilman for training me in EEG recordings and processing and Abbas Ali for his help as a fellow student on this project. 


\section{References}

Bieniek, M.M., Frei, L.S., \& Rousselet, G.A. (2013) Early ERPs to faces: Aging, luminance, and individual differences. Front. Psychol., 4.

Delorme, A. \& Makeig, S. (2004) EEGLAB: An open source toolbox for analysis of singletrial EEG dynamics including independent component analysis. J. Neurosci. Methods, $134,9-21$.

Dougherty, B.E., Flom, R.E., \& Bullimore, M.A. (2005) An evaluation of the Mars Letter Contrast Sensitivity Test. Optom. Vis. Sci., 82, 970-975.

Ebner, N.C., Riediger, M., \& Lindenberger, U. (2010) FACES--a database of facial expressions in young, middle-aged, and older women and men: development and validation. Behav. Res. Methods, 42, 351-362.

Gazzaley, A., Clapp, W., Kelley, J., McEvoy, K., Knight, R.T., \& D’Esposito, M. (2008) Age-related top-down suppression deficit in the early stages of cortical visual memory processing. Proc. Natl. Acad. Sci., 105, 13122-13126.

Gill, D., DeBruine, L., Jones, B., \& Schyns, P. (2015) Bubble-Warp: a New Approach to the Depiction of High-Level Mental Representation. J. Vis., 15, 420.

Gosselin, F. \& Schyns, P.G. (2001) Bubbles: A technique to reveal the use of information in recognition tasks. Vision Res., 41, 2261-2271.

Grady, C.L. (2008) Cognitive neuroscience of aging. Ann. N. Y. Acad. Sci., 1124, 127-144.

Grady, C.L., Maisog, J.M., Horwitz, B., Ungerleider, L.G., Mentis, M.J., Salerno, J. a, Pietrini, P., Wagner, E., \& Haxby, J. V (1994) Age-related changes in cortical blood flow activation during visual processing of faces and location. J. Neurosci., 14, 14501462. 
Gunning-Dixon, F.M., Gur, R.C., Perkins, A.C., Schroeder, L., Turner, T., Turetsky, B.I., Chan, R.M., Loughead, J.W., Alsop, D.C., Maldjian, J., \& Gur, R.E. (2003) Age-related differences in brain activation during emotional face processing. Neurobiol. Aging, 24, $285-295$.

Haxby, J. V., Hoffman, E.A., \& Gobbini, M.I. (2002) Human neural systems for face recognition and social communication. Biol. Psychiatry, 51, 59-67.

Iidaka, T., Okada, T., Murata, T., Omori, M., Kosaka, H., Sadato, N., \& Yonekura, Y. (2002) Age-related differences in the medial temporal lobe responses to emotional faces as revealed by fMRI. Hippocampus., 12, 352-362.

Ince, R.A.A., Giordano, B.L., Kayser, C., Rousselet, G.A., Gross, J., \& Schyns, P.G. (2016) A statistical framework for neuroimaging data analysis based on mutual information estimated via a Gaussian copula. bioRxiv, 1-53.

Jaworska, K., Yi, F., Ince, R.A.A., Schyns, P.G., \& Rousselet, G.A. (2016) Processing of the same face features is delayed by $40 \mathrm{~ms}$ and weaker in healthy ageing. Manuscript in preparation.

Kayser, J. \& Tenke, C.E. (2006) Principal components analysis of Laplacian waveforms as a generic method for identifying ERP generator patterns: I. Evaluation with auditory oddball tasks. Clin. Neurophysiol., 117, 348-368.

Mouchetant-Rostaing, Y. \& Giard, M.H. (2003) Electrophysiological correlates of age and gender perception on human faces. J. Cogn. Neurosci., 15, 900-910.

Mouchetant-Rostaing, Y., Giard, M.H., Bentin, S., Aguera, P.E., \& Pernier, J. (2000) Neurophysiological correlates of face gender processing in humans. Eur. J. Neurosci., $12,303-310$. 
Nasreddine, Z.S., Phillips, N.A., Bedirian, V., Charbonneau, S., Whitehead, V., Collin, I., Cummings, J.L., \& Chertkow, H. (2005) The Montreal Cognitive Assessment, MoCA: a brief screening tool for mild cognitive impairment. J. Am. Geriatr. Soc., 53, 695-699.

Reddy, L., Wilken, P., \& Koch, C. (2004) Face-gender discrimination is possible in the nearabsence of attention. J. Vis., 4, 106-117.

Rousselet, G.A., Gaspar, C.M., Pernet, C.R., Husk, J.S., Bennett, P.J., \& Sekuler, A.B. (2010) Healthy aging delays scalp EEG sensitivity to noise in a face discrimination task. Front. Psychol., 1.

Rousselet, G.A., Ince, R.A.A., van Rijsbergen, N.J., \& Schyns, P.G. (2014) Eye coding mechanisms in early human face event-related potentials. J. Vis., 14, 1-24.

Ruffman, T., Henry, J.D., Livingstone, V., \& Phillips, L.H. (2008) A meta-analytic review of emotion recognition and aging: Implications for neuropsychological models of aging. Neurosci. Biobehav. Rev., 32, 863-881.

Santini, Z.I., Fiori, K.L., Feeney, J., Tyrovolas, S., Haro, J.M., \& Koyanagi, A. (2016) Social relationships, loneliness, and mental health among older men and women in Ireland: A prospective community-based study. J. Affect. Disord., 204, 59-69.

Schyns, P.G., Bonnar, L., \& Gosselin, F. (2002) Show me the features! Understanding recognition from the use of visual information. Psychol. Sci. a J. Am. Psychol. Soc. / APS, 13, 402-409.

Schyns, P.G., Thut, G., \& Gross, J. (2011) Cracking the code of oscillatory activity. PLoS Biol., 9.

Smith, M.L., Gosselin, F., \& Schyns, P.G. (2004) Receptive fields for flexible face categorizations. Psychol. Sci., 15, 753-761. 
Smith, M.L., Gosselin, F., \& Schyns, P.G. (2007) From a face to its category via a few information processing states in the brain. Neuroimage, 37, 974-984.

Sullivan, S., Ruffman, T., \& Hutton, S.B. (2007) Age differences in emotion recognition skills and the visual scanning of emotion faces. J. Gerontol. B. Psychol. Sci. Soc. Sci., 62, P53-P60.

Tenke, C.E. \& Kayser, J. (2012) Generator localization by current source density (CSD): implications of volume conduction and field closure at intracranial and scalp resolutions. Clin. Neurophysiol., 123, 2328-2345.

Voss, M.W., Erickson, K.I., Chaddock, L., Prakash, R.S., Colcombe, S.J., Morris, K.S., Doerksen, S., Hu, L., McAuley, E., \& Kramer, A.F. (2008) Dedifferentiation in the visual cortex: an fMRI investigation of individual differences in older adults. Brain Res., 1244, 121-131. 


\section{Supporting Information}

Fig. S1. Each participant's individual classification images of MI(PIX, ACC) and MI(PIX, $R T)$. PO1 refers to the older participant, with all other classification images referring young participants.
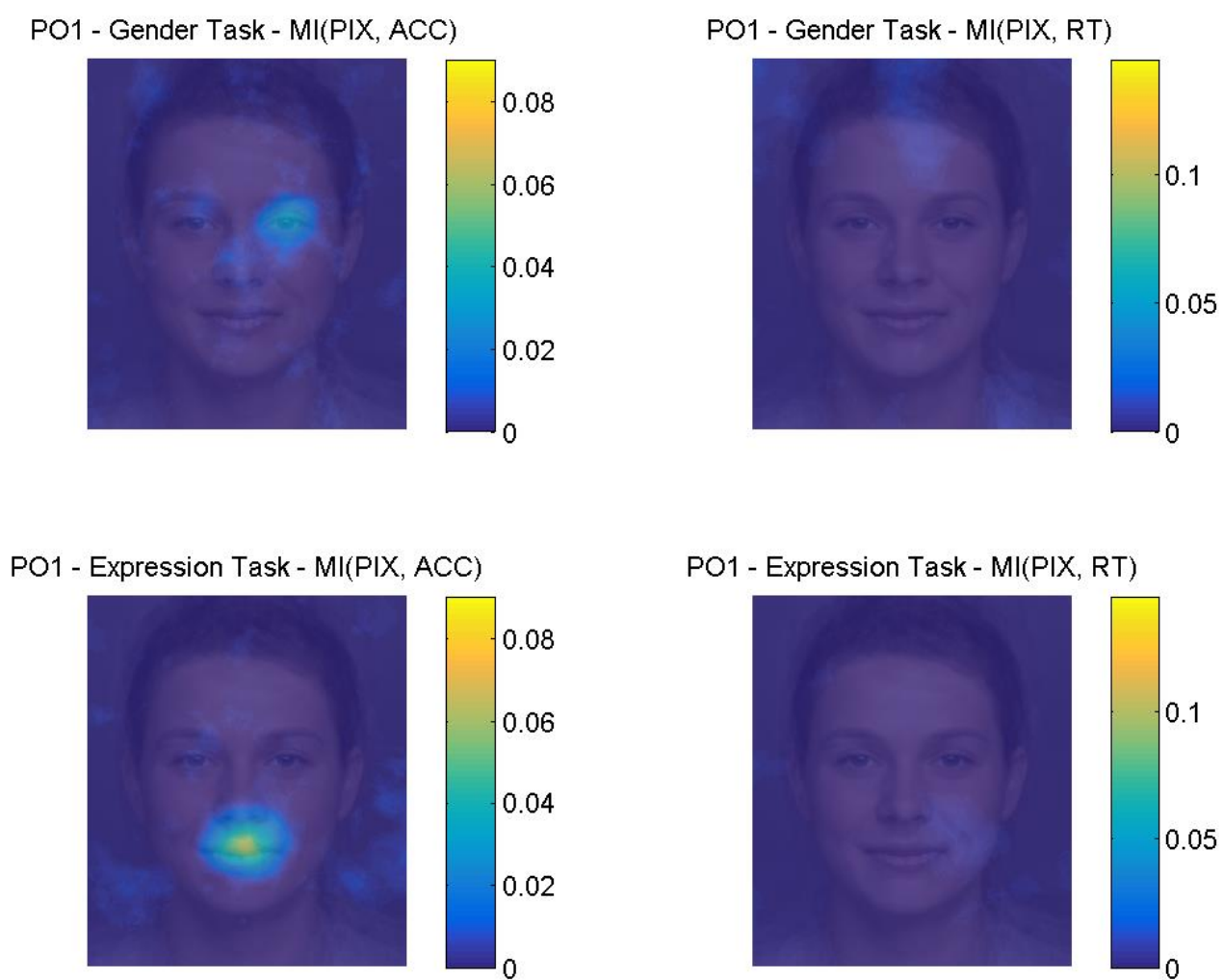


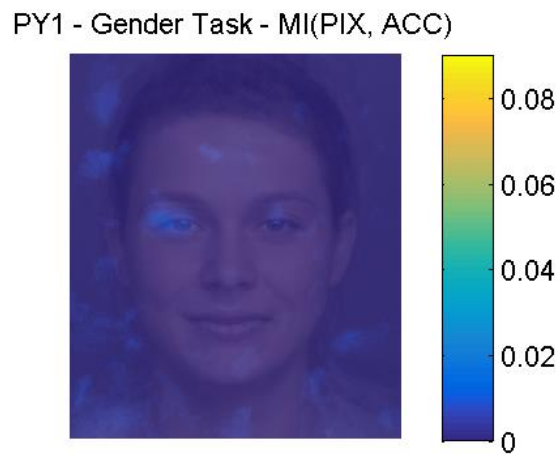

PY1 - Expression Task - MI(PIX, ACC)

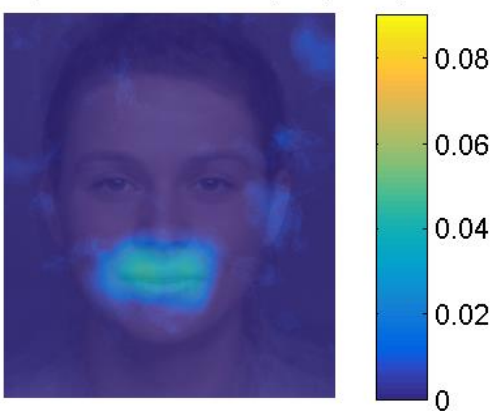

PY2 - Gender Task - MI(PIX, ACC)

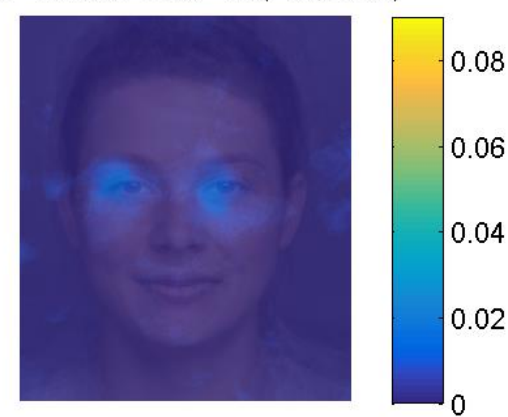

PY2 - Expression Task - MI(PIX, ACC)

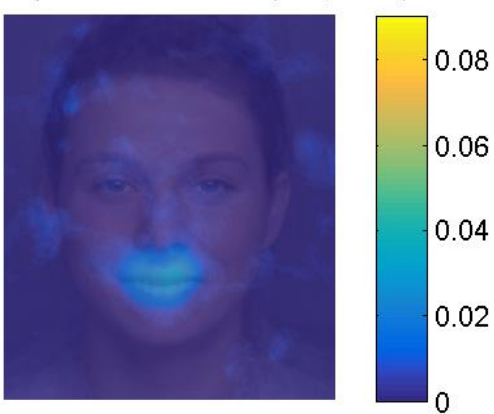

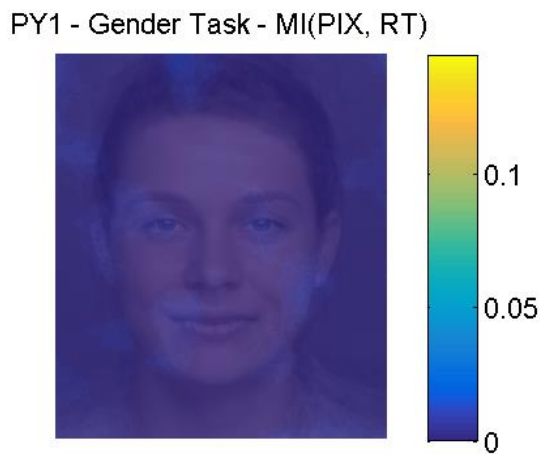

PY1 - Expression Task - MI(PIX, RT)

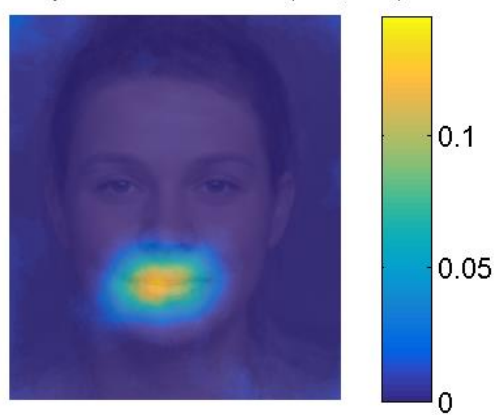

PY2 - Gender Task - MI(PIX, RT)

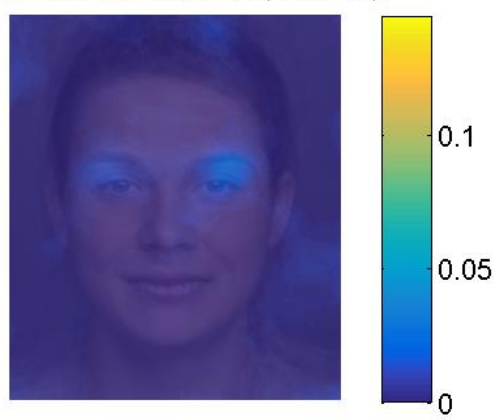

PY2 - Expression Task - MI(PIX, RT)

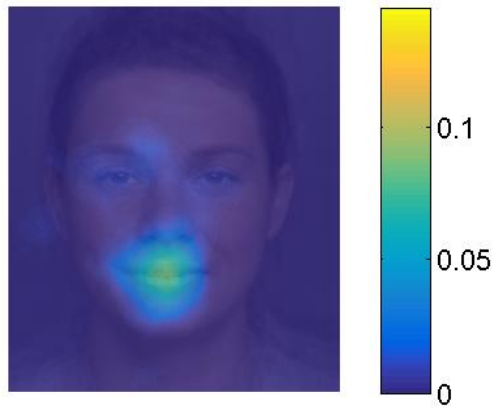




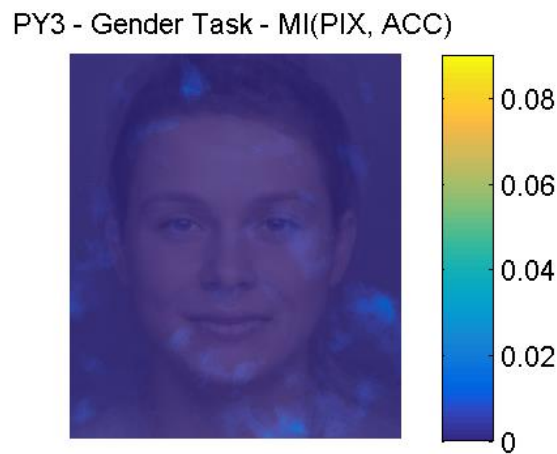

PY3 - Expression Task - MI(PIX, ACC)

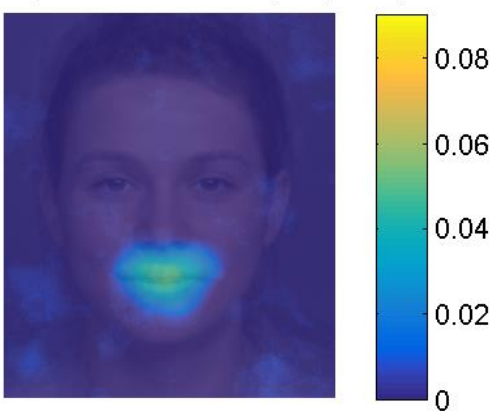

PY4 - Gender Task - MI(PIX, ACC)

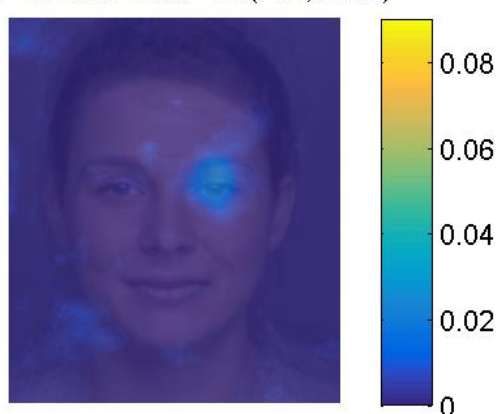

PY4 - Expression Task - MI(PIX, ACC)

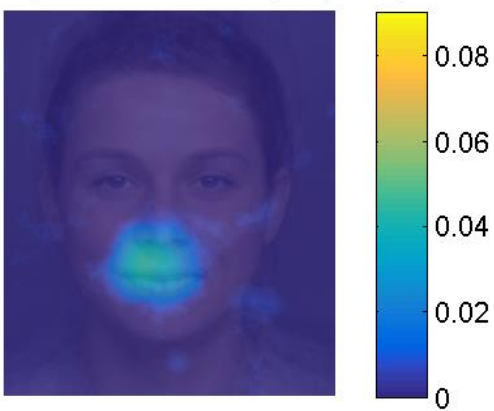

PY3 - Gender Task - MI(PIX, RT)

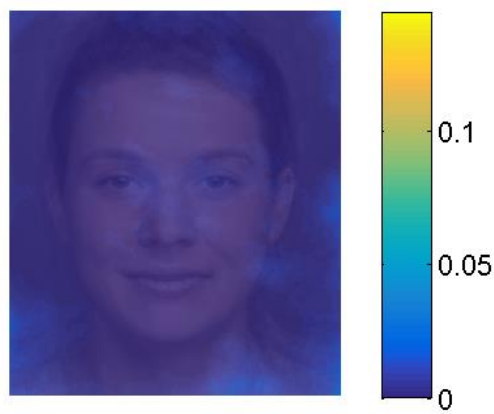

PY3 - Expression Task - MI(PIX, RT)

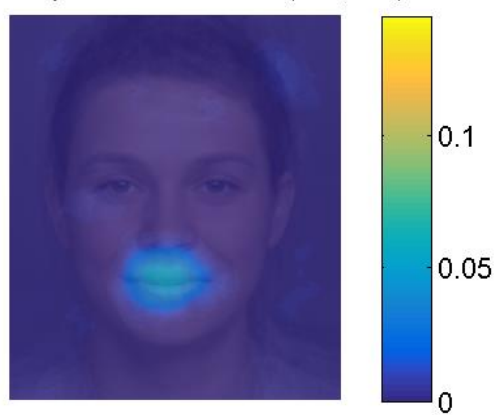

PY4 - Gender Task - MI(PIX, RT)

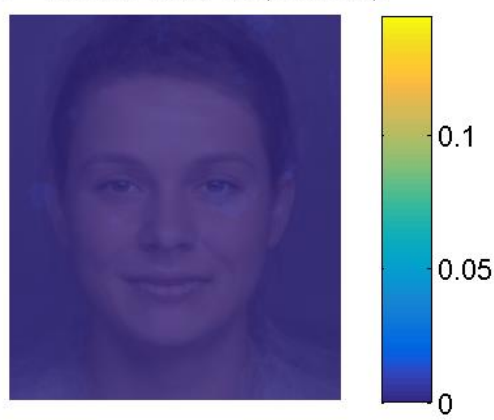

PY4 - Expression Task - MI(PIX, RT)

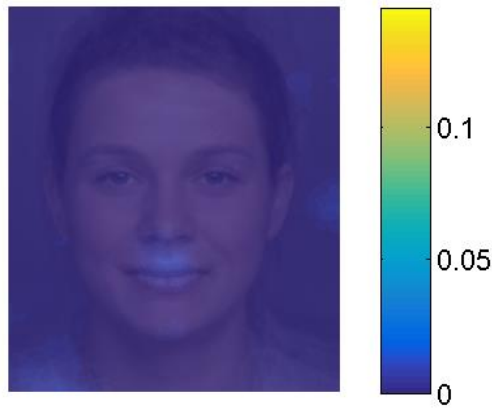




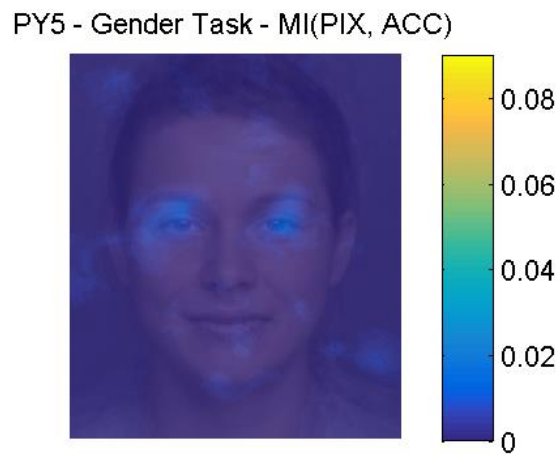

PY5 - Expression Task - MI(PIX, ACC)
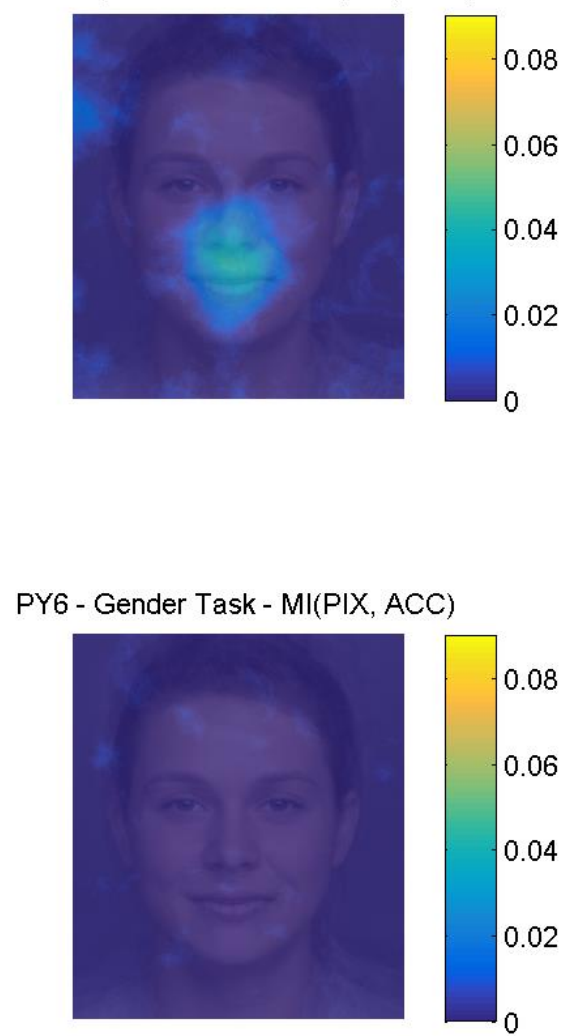

PY6 - Expression Task - MI(PIX, ACC)

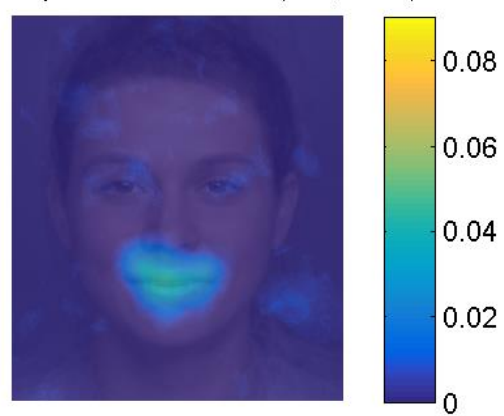

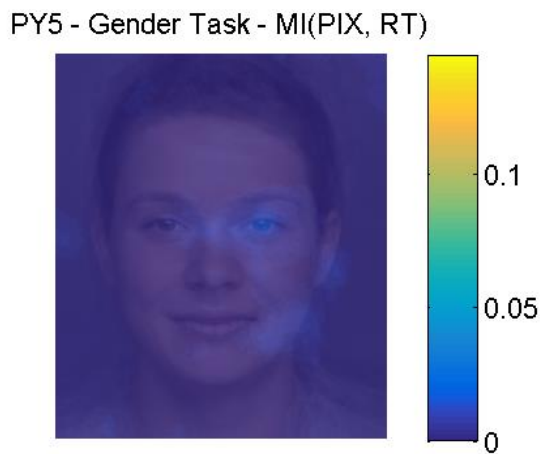

PY5 - Expression Task - MI(PIX, RT)

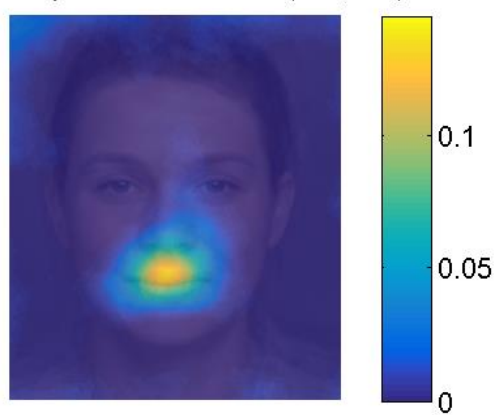

PY6 - Gender Task - MI(PIX, RT)

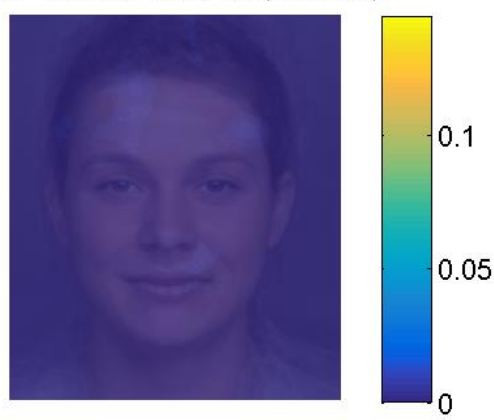

PY6 - Expression Task - MI(PIX, RT)

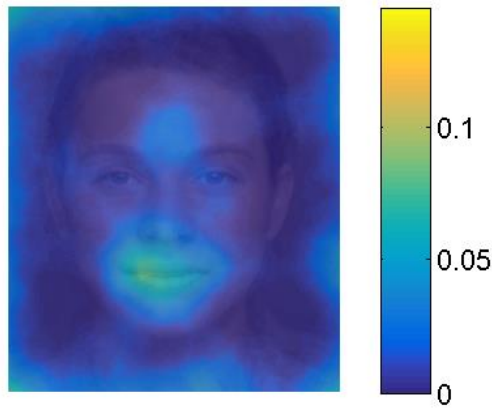


PY7 - Gender Task - MI(PIX, ACC)

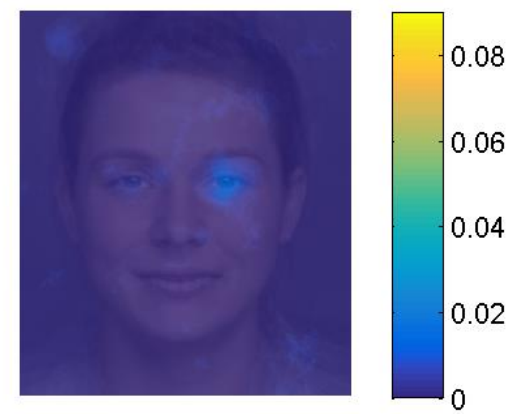

PY7 - Expression Task - MI(PIX, ACC)

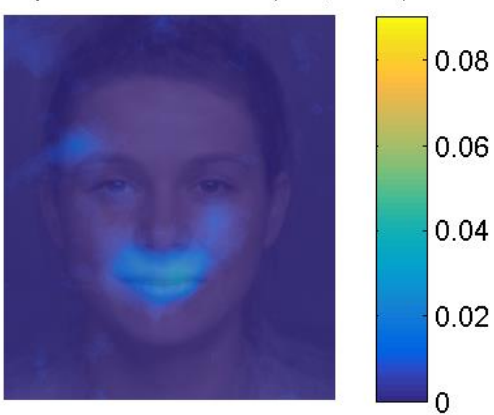

PY8 - Gender Task - MI(PIX, ACC)

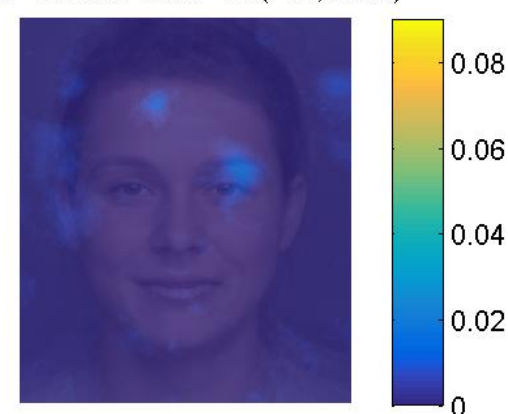

PY8 - Expression Task - MI(PIX, ACC)

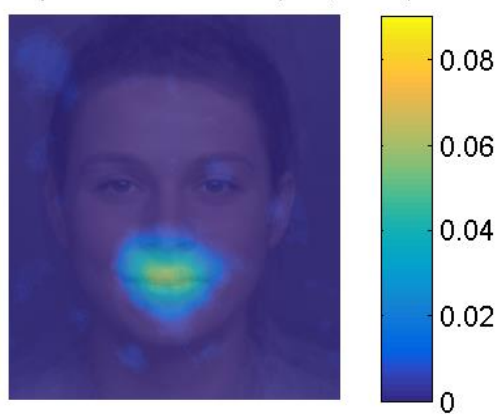

PY7 - Gender Task - MI(PIX, RT)

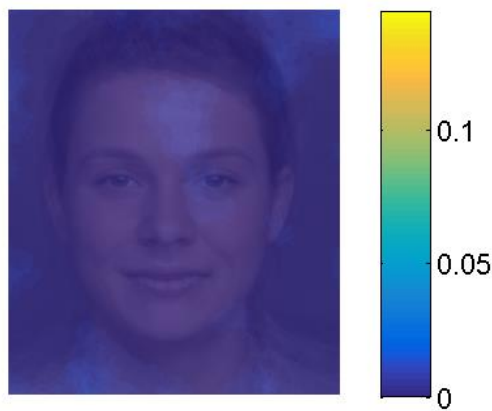

PY7 - Expression Task - MI(PIX, RT)

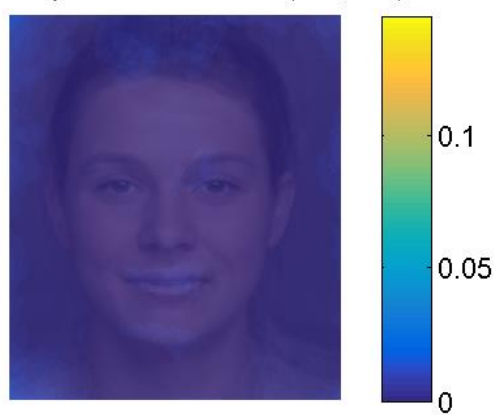

PY8 - Gender Task - MI(PIX, RT)

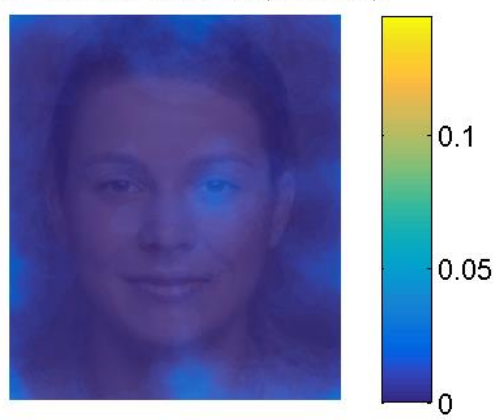

PY8 - Expression Task - MI(PIX, RT)

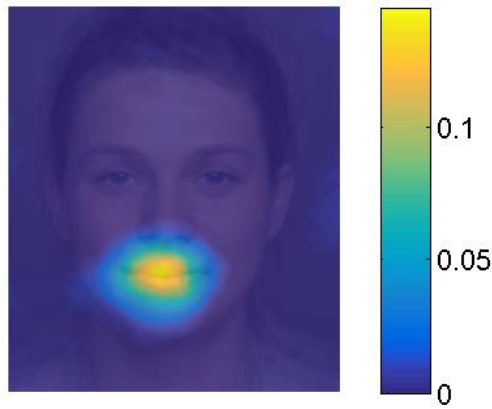




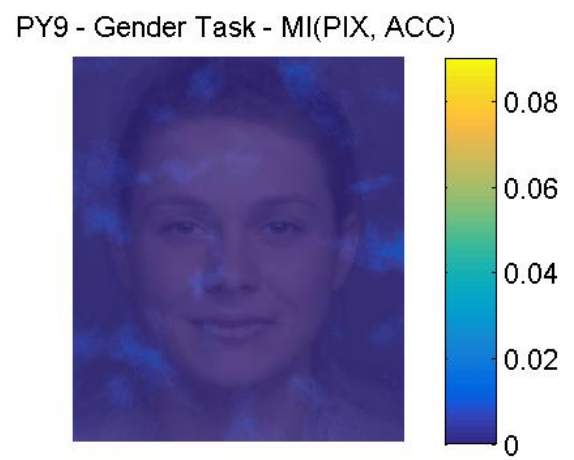

PY9 - Expression Task - MI(PIX, ACC)

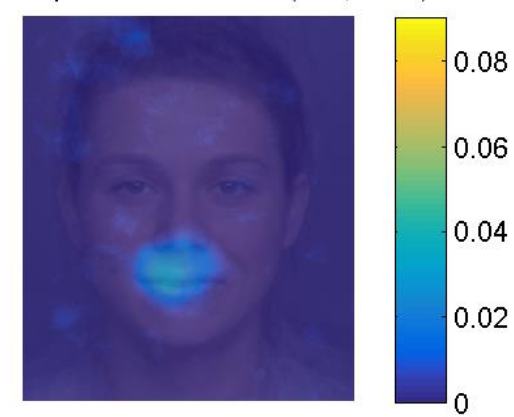

PY10 - Gender Task - MI(PIX, ACC)

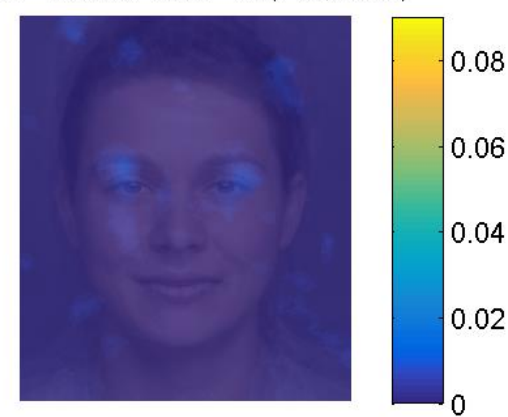

PY10 - Expression Task - MI(PIX, ACC)

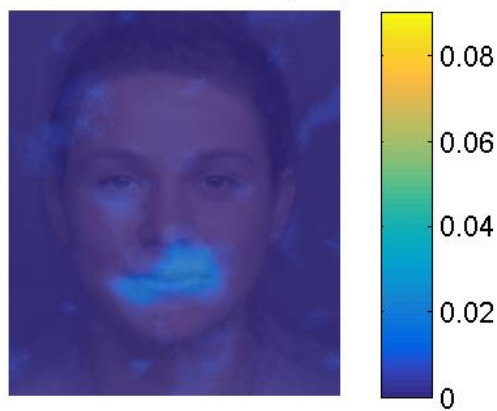

PY9 - Gender Task - MI(PIX, RT)

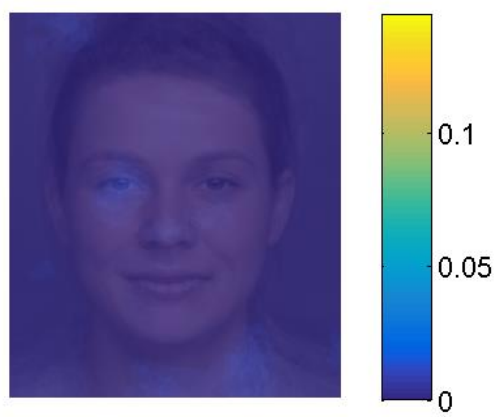

PY9 - Expression Task - MI(PIX, RT)

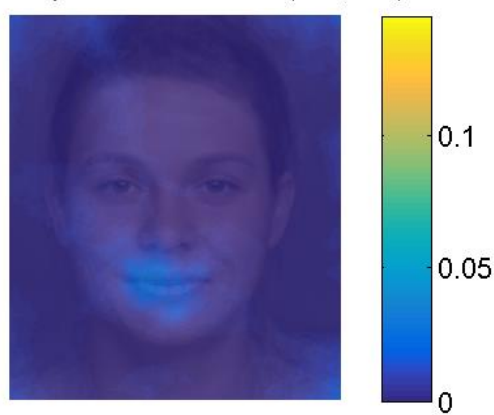

PY10 - Gender Task - MI(PIX, RT)

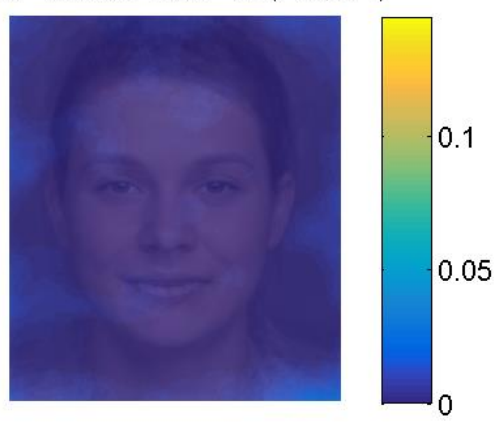

PY10 - Expression Task - MI(PIX, RT)

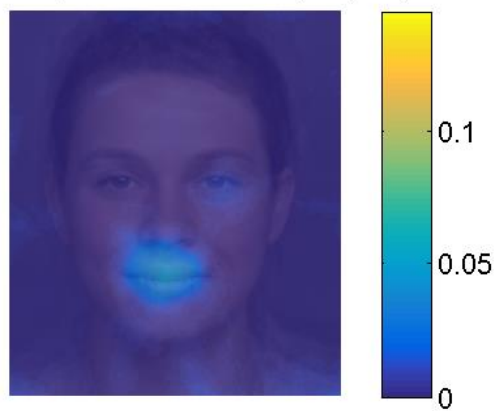




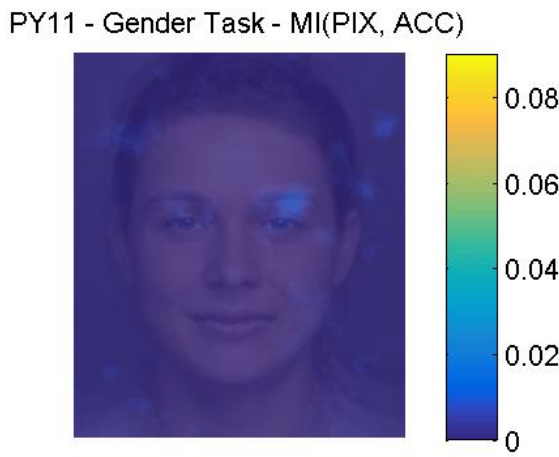

PY11 - Expression Task - MI(PIX, ACC)

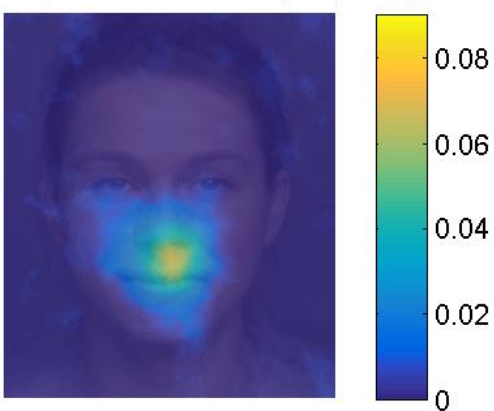

PY12 - Gender Task - MI(PIX, ACC)

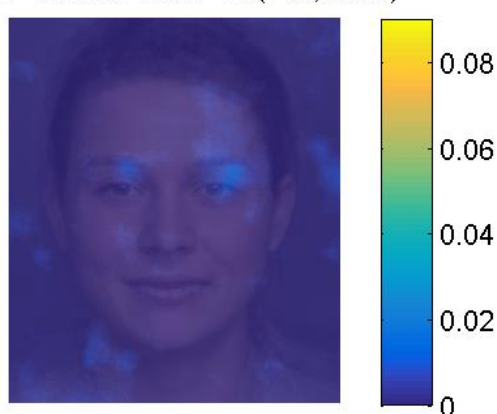

PY12 - Expression Task - MI(PIX, ACC)

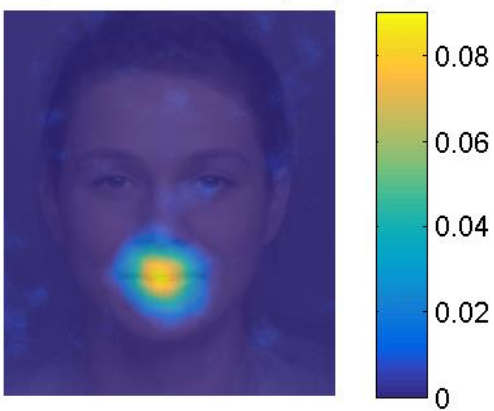

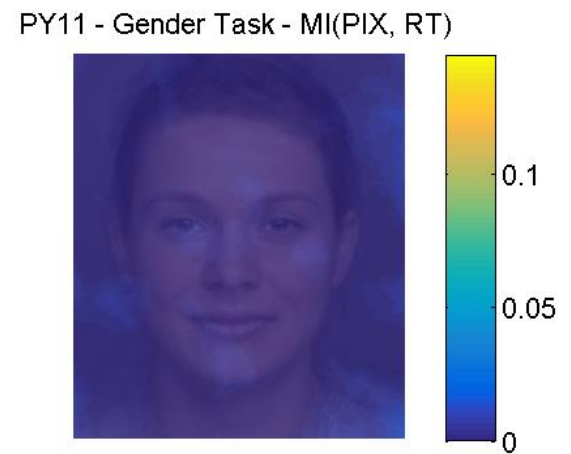

PY11 - Expression Task - MI(PIX, RT)

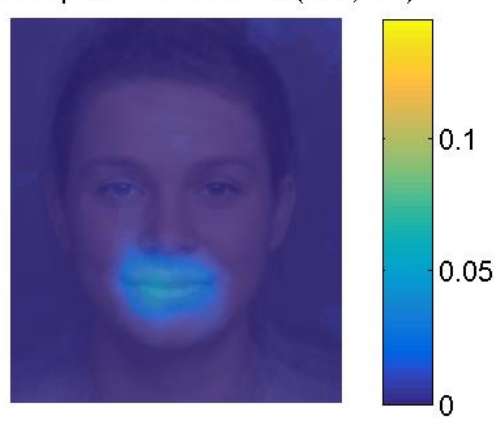

PY12 - Gender Task - MI(PIX, RT)

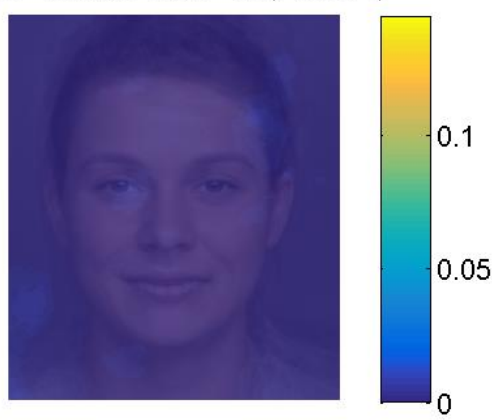

PY12 - Expression Task - MI(PIX, RT)

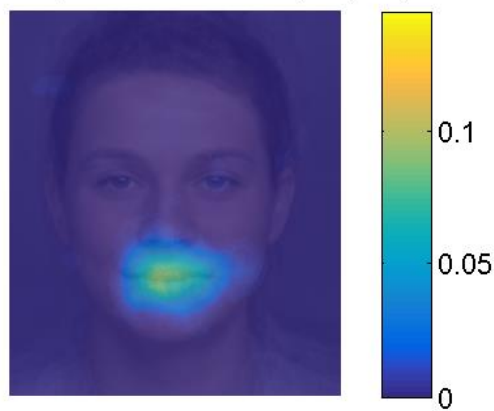




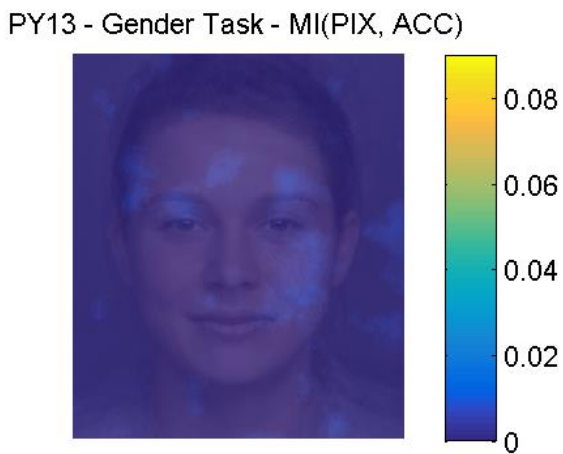

PY13 - Expression Task - MI(PIX, ACC)

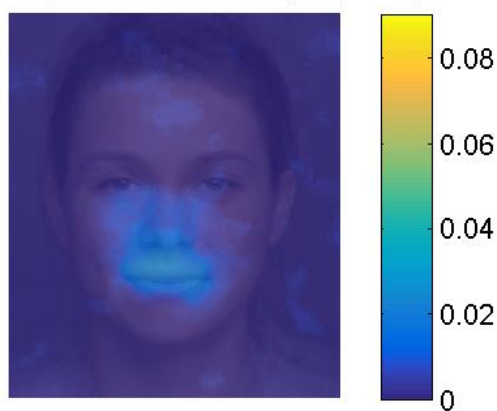

PY13 - Gender Task - MI(PIX, RT)

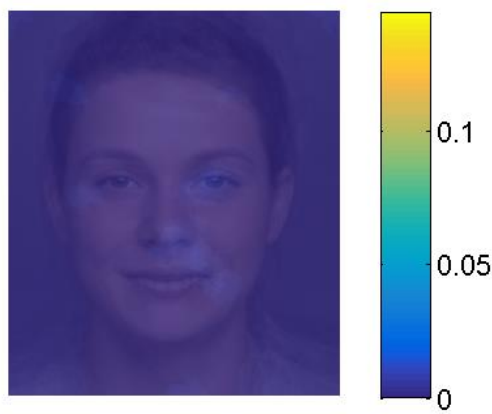

PY13 - Expression Task - MI(PIX, RT)

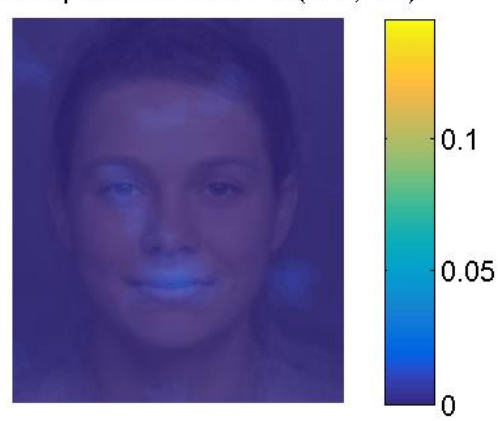

